\title{
RICCI FLOW, EINSTEIN METRICS AND SPACE FORMS
}

\author{
RUGANG YE
}

\begin{abstract}
The main results in this paper are: (1) Ricci pinched stable Riemannian metrics can be deformed to Einstein metrics through the Ricci flow of R. Hamilton; (2) (suitably) negatively pinched Riemannian manifolds can be deformed to hyperbolic space forms through Ricci flow; and (3) $L^{2}$-pinched Riemannian manifolds can be deformed to space forms through Ricci flow.
\end{abstract}

\section{INTRODUCTION AND MAIN RESULTS}

Einstein metrics on a compact manifold $M$ of dimension $n \geq 3$ are characterized as critical points of the normalized total scalar curvature functional S on the space $\mathscr{M}$ of all (Riemannian) metrics on $M$. A natural procedure to construct Einstein metrics is therefore to deform an initial metric along the gradient flow of $\mathbf{S}$. The explicit formula for $\mathbf{S}$ is this: for $g \in \mathscr{M}$,

$$
\mathbf{S}(g)=\frac{1}{V(g)^{(n-2) / n}} \int_{M} R_{g} d v_{g}
$$

where $d v_{g}$ is the volume form of $g, V(g)=\int_{M} d v_{g}$, and $R_{g}$ denotes the scalar curvature function of $g$. Simple computations [Sc] show that the gradient of $\mathbf{S}$ at $g$ is given by

$$
-V(g)^{(2-n) / n}\left(R c_{g}-\frac{1}{2} R_{g} g+\frac{n-2}{2 n} \bar{R}_{g} g\right),
$$

where $R c_{g}$ denotes the Ricci tensor of $g$ and $\bar{R}_{g}=V(g)^{-1} \int_{M} R_{g} d v_{g}$. Assuming w.l.o.g. $V(g)=1$ at time $t=0$ we can then write the gradient flow in the following way:

$$
\frac{\partial g}{\partial t}=-T_{g}+\frac{n-2}{2 n} \delta R_{g} g
$$

Here $T_{g}=R c_{g}-R_{g} g / n$ is the traceless Ricci tensor of $g$ and $\delta R_{g}=R_{g}-\bar{R}_{g}$. Along this flow the functional $\mathbf{S}$ would be increased. However, one observes [Sc] that an Einstein metric always minimizes $\mathbf{S}$ in its conformal class. Consequently one has to reverse the sign of the second term on the right-hand side of (0.1), which is the conformal component of the gradient. We keep the sign of

Received by the editors November 14, 1990 and, in revised form, May 20, 1991.

1991 Mathematics Subject Classification. Primary 53C20; Secondary 53C21, 53C25, 58G11, $58 \mathrm{G} 25$.

Key words and phrases. Ricci flow, Einstein metrics, stability, space forms.

Partially supported by NSF grant no. DMS87-03537. 
the orthogonal component $-T_{g}$ of the gradient, because an Einstein metric infinitesimally maximizes $\mathbf{S}$ in all, except possibly a finite dimensional subspace of, directions orthogonal to its conformal class [Sc]. Thus we are lead to the following rectified gradient flow:

$$
\frac{\partial g}{\partial t}=-T_{g}-\frac{n-2}{2 n} \delta R_{g} g .
$$

For convenience, one can change the scale of the conformal component, e.g., replace $(n-2) / 2 n$ by $1 / n$. Then one arrives at the Ricci flow of Hamilton [Ha1]:

$$
\frac{\partial g}{\partial t}=-2 T_{g}-\frac{2 \delta R_{g}}{n} g \quad \text { or } \quad \frac{\partial g}{\partial t}=-2 R c_{g}+\frac{2 \bar{R}_{g}}{n} g .
$$

(The factor 2 is added to simplify computations.) Note that there is a certain restriction to the scale factor of the conformal component in order to ensure short time existence of the flow, see [Ni]. On the other hand, the scale factors do not affect the structure of the equation concerning long time behavior.

The Ricci flow $(0.2)$ has been successfully used by Hamilton to study 3manifolds of positive Ricci curvature [Ha1] and 4-manifolds of positive curvature operator [Ha2]. He showed that these manifolds can be deformed to spherical space forms through the Ricci flow, i.e., the Ricci flow starting at such manifolds exists for all time and converges smoothly to spherical space forms as the time goes to infinity. Following Hamilton's approach, Huisken [Hu], Margerin [Ma], and Nishikawa [Ni] proved that pointwise (sufficiently) pinched manifolds of positive sectional curvature can be deformed to spherical space forms through the Ricci flow. (The topological implication that such manifolds are diffeomorphic to quotients of spheres had earlier been verified by Ruh [Ru] using a completely different method. The Ricci flow method produces much better pinching constants.)

So far a short account of the Ricci flow. Now we explain the content of this paper. Our first purpose is constructing Einstein metrics through the Ricci flow under an Einstein or Ricci pinching condition. Roughly speaking, for a given stable metric $g$, if the $L^{2}$ norm of the traceless Ricci tensor $T_{g}$ is small relative to suitable geometric quantities, then one can deform $g$ to an Einstein metric through the Ricci flow. The concept "stability" is defined as follows. (Henceforth we omit the subscript $g$ in notations for geometric quantities associated with $g$.) For a given $g \in \mathscr{M}$ let $Q$ be the quadratic form

$$
Q(h)=\int|\nabla h|^{2}-2 \int R m(h) \cdot h+\int T(h) \cdot h
$$

on symmetric 2-tensors $h=h_{i j} d x^{i} d x^{j}$. Here $R m=R_{i j k l} d x^{i} d x^{j} d x^{k} d x^{l}$ denotes the Riemann curvature tensor (such that $R_{1212}>0$ for standard spheres), $R m(h)_{i j}=R_{i p j q} h^{p q}, T(h)_{i j}=T_{i k} h^{k} j$ (as usual, $h^{i j}=g^{i p} g^{j q} h_{p q}, h_{j}^{i}=g^{i k} h_{k j}$ and the summation convention is used), and the dot denotes inner product. (Of course, the integrals are defined on $M$ and with respect to the volume form of g.) We shall call $Q$ "Einstein form".

Definition 1. The Einstein eigenvalues of $g$ are the eigenvalues of the operator $L$ acting on traceless symmetric 2-tensors,

$$
L h=-\Delta h-2 R m(h)+T(h) .
$$


Throughout this paper, $\Delta$ denotes the trace Laplacian. We denote the minimum Einstein eigenvalue by $\lambda_{e}$. Clearly $\lambda_{e}$ equals the minimum of $Q(h)$ over all traceless symmetric 2-tensors $h$ with $\int|h|^{2}=1$. If $\lambda_{e}>0$, then we say that $g$ is stable.

The operator $L$ is the Euler-Lagrange operator of $Q$ and differs from the Lichnerowitz Laplacian [Be, 1.143] by a zeroth order term involving the Ricci tensor. One finds that, e.g., partially Ricci pinched metrics of negative sectional curvatures as well as (Riemann) pinched metrics of nonzero scalar curvature are stable (Propositions 3 and 4).

Now we state

Theorem 1. Let $(M, g)$ be a closed Riemannian manifold of dimension $n \geq 3$ satisfying $d^{2}\|R m\|_{C^{0}} \leq \Lambda, d^{2} \lambda_{e} \geq \sigma$ and the pinching condition either

$$
f|T|^{2} \leq \varepsilon_{1}(n, \Lambda, \sigma) \frac{1}{d^{4}}
$$

or

$$
f|T|^{2} \leq \varepsilon_{2}(n, \Lambda, \sigma)\|R m\|_{C^{0}}^{2}
$$

for some positive numbers $\Lambda, \sigma$, where $d=$ diameter, $f=(1 / V) \int$, and

$$
\begin{aligned}
& \varepsilon_{1}(n, \Lambda, \sigma)=\frac{1}{c(n)} e^{-c(n) \sqrt{\Lambda}} \min \left(1, \sigma^{8(3 n+8)}\right), \\
& \varepsilon_{2}(n, \Lambda, \sigma)=\frac{1}{c(n)} e^{-c(n) \sqrt{\Lambda}} \min \left(1,\left(\frac{\sigma}{\Lambda}\right)^{8(3 n+8)}\right)
\end{aligned}
$$

with a certain positive constant $c(n)$ depending only on $n$. Then $g$ can be deformed to an Einstein metric through the Ricci flow. In particular, $M$ supports Einstein metrics. (Note that $\|R m\|_{C^{0}}$ is equivalent to the maximal absolute value of sectional curvatures.)

Remark 1. The bound on $\|R m\|_{C^{0}}$ can be replaced by a bound on $\int|R m|^{p}$ with $p>n / 2$ and a pointwise lower bound on the Ricci curvature.

Remark 2. The theorem still holds if the condition $d^{2} \lambda_{e} \geq \sigma$ is replaced by $\lambda_{e} /\|R m\|_{C^{0}} \geq \sigma$ and the first pinching condition is omitted (the number $\varepsilon_{2}$ in the second pinching condition should be replaced by $\varepsilon_{1}$ ).

Remark 3. There have been works on Ricci pinching based on Gromov's compactness theorem, see, e.g., [Gao]. Besides the independent interest of deformation by the Ricci flow, one should note that no lower bound on injectivity radius (or related quantity) is assumed here and hence the compactness theory is not applicable.

A special (analytic) consequence of this theorem is the following stability theorem for the Ricci flow: the Ricci flow starting near a stable Einstein metric always converges to it. This reveals the strong variational structure of the Ricci flow. Another special corollary is that Ricci pinched manifolds of negative sectional curvatures can be deformed to Einstein manifolds.

Corollary. Let $(M, g)$ be a closed Riemannian manifold of dimension $n \geq 3$ such that $-1 \geq K^{+} \geq K^{-} \geq-\Lambda$ and $\|T\|_{C^{0}} \leq e^{-c(n) \Lambda(d+1)} / c(n)$ for some number $\Lambda \geq 1$, where $c(n)$ is a certain positive constant depending only on $n$, 
and $\mathrm{K}^{+}, \mathrm{K}^{-}$denote respectively the maximal and minimal sectional curvatures. Then $g$ can be deformed to an Einstein metric through the Ricci flow. Along the flow and in the limit, Ricci curvatures remain negative.

In the proof of Theorem 1 we adopt a new approach to the study of convergence of the Ricci flow. Hamilton's approach essentially consists of (ingeniously) applying pointwise parabolic maximum principles to various curvature quantities and thereupon establishing that along the flow the Riemann curvature tensor becomes more and more pinched and the scalar curvature approaches a constant. This approach is very powerful, but depends on positivity of curvatures, mainly for the reason of the maximum principles. The approach of the present paper follows the scheme proposed by Min-Oo [Mi]: via the weak maximum principle of Moser [Mo], $L^{2}$ estimates of curvature quantities can be translated into $C^{0}$ estimates and hence the convergence of the Ricci flow is reduced to establishing $L^{2}$ decay of the traceless Ricci tensor $T$. Unfortunately, the crucial $L^{2}$ decay argument in [Mi] is not correct and the claimed theorem there (stating that almost Einstein metrics of negative Ricci curvature can be deformed to Einstein metrics) remains open. The vital fact which was overlooked in [Mi] is that the projection $\operatorname{Pr}$ defined in (2.11) in [Mi] is not orthogonal, hence the equation

$$
\left\langle\operatorname{Pr}\left(\frac{\partial}{\partial t} R m\right), Z\right\rangle=\left\langle\frac{\partial}{\partial t} R m, Z\right\rangle
$$

does not hold ( $Z$ is the traceless Ricci curvature tensor of type $(1,3)$, see [Mi]). This equation is essential for the proof of the crucial estimate of (2.21) in [Mi]. We are unable to find a correct proof of this estimate and believe that it does not hold in general. We remark that the difference of the two sides of the above wrong equation contains exactly what is hard to handle. Our observation is that the desired $L^{2}$ decay of $T$ can be derived from stability. We would like to note that the issue of preserving stability along the flow is rather delicate. This is true even for short time. For long time, stability and convergence are intertwined: they depend on each other and will be proven simultaneously. Because we only establish a certain partial stability along the flow, we do not know whether the limit Einstein metric is stable. We point out that this is the case, provided that one additionally imposes a Hölder bound on the covariant derivatives of the Ricci tensor of the initial metric. Such a bound is not so natural though. We also notice that in Theorem 1 we can actually replace the stability condition by a weaker condition, namely $H$-stability (i.e., replace $\lambda_{e}$ by the number $\lambda^{H}$ below).

Definition 2. Consider a metric $g$ (on a manifold $M$ ). For $H>0$ set

$$
\begin{aligned}
& \lambda^{H}=\inf \left\{Q(h) / \int|h|^{2}: h\right. \text { is a traceless symmetric 2-tensor } \\
& \text { with } \left.\|h\|_{C^{0}}^{2} \leq H \int|h|^{2} / \int|T|^{2}\right\} .
\end{aligned}
$$

If $\lambda^{H}>0$, then we say that $g$ is $H$-stable. ( $H$-stability coincides with stability provided that $g$ is Einstein. Also, stability can be considered as the special case $H=\infty$ of $H$-stability.) 
Our second topic is to extend the curvature pinching and deformation theorem of Huisken, Margerin, and Nishikawa mentioned before. We prove

Theorem 2. For given $n \geq 3$ and $C>0$ there exists an $\varepsilon>0$ such that the following is true. If a closed n-dimensional Riemannian manifold $(M, g)$ satisfies the pinching condition $-1 \geq K^{+} \geq K^{-} \geq-1-\varepsilon$ and one of the boundedness conditions below, then it can be deformed to a hyperbolic space form through the Ricci flow

(1) $d \leq C$,

(2) $V \leq C, n \neq 3$,

(3) $|\chi| \leq C, n$ is even, where $\chi$ is the Euler characteristic. Moreover, sectional curvatures remain negative along the flow.

The main point in this theorem is the Ricci deformation, because the topological implication that $M$ is diffeomorphic to a hyperbolic space form was known (due to Gromov [Gr]). In the following two theorems we deal with $L^{2}$ curvature pinching. There are three different ways of measuring $L^{2}$-pinching of curvature: in terms of $\int|\widetilde{R} m|^{2}, \int\left|R^{ \pm} m\right|^{2}$, or $\int|\stackrel{R}{R} m|^{2}$, where

$$
\begin{gathered}
(\tilde{R} m)_{i j k l}=R_{i j k l}-\frac{\bar{R}}{n(n-1)}\left(g_{i k} g_{j l}-g_{i l} g_{j k}\right), \\
\left(R^{ \pm} m\right)_{i j k l}=R_{i j k l} \mp \frac{1}{n(n-1)}\left(g_{i k} g_{j l}-g_{i l} g_{j k}\right),
\end{gathered}
$$

and

$$
\stackrel{\circ}{R}_{i j k l} \equiv(\stackrel{\circ}{R} m)_{i j k l}=R_{i j k l}-\frac{R}{n(n-1)}\left(g_{i k} g_{j l}-g_{i l} g_{j k}\right) .
$$

$(\stackrel{\circ}{R} m$ is called "concircular curvature tensor" and naturally arises in the Weyl decomposition of curvature tensors.) The first two measure global pinching in $L^{2}$ norm, while the third, the weakest of the three, measures pointwise pinching in $L^{2}$ norm. (Note that smallness of $\int|\stackrel{R}{R} m|^{2}$ does not imply smallness of $\int|\delta R|^{2}$.) We first consider $L^{2}$-global pinching.

Theorem 3. For given $n \geq 3$ and $\Lambda>0$ there exists an $\varepsilon>0$ with the following property. If a closed Riemannian manifold $(M, g)$ of dimension $n$ satisfies $d^{2}\|R m\|_{C^{0}} \leq \Lambda, f|\widetilde{R} m|^{2} \leq \varepsilon \bar{R}^{2}$, then it can be deformed to a space form through the Ricci flow. In particular, $M$ is diffeomorphic to a space form. The curvature sign of the limit space form coincides with that of $\bar{R}$.

Next we treat $L^{2}$-pointwise pinching.

Theorem 4. For given $n \geq 3, \Lambda>0$ there exists an $\varepsilon>0$ with the following property. If a closed Riemannian manifold $(M, g)$ of dimension $n$ satisfies $d^{2}\|R m\|_{C^{0}} \leq \Lambda, f|\stackrel{\circ}{R} m|^{2} \leq \varepsilon \bar{R}^{4} /\|R m\|_{C^{0}}^{2}$ or $\leq \varepsilon d^{4} \bar{R}^{4}$ and $\bar{R}<0$, then it can be deformed to a hyperbolic space form through the Ricci flow.

In case $\bar{R}>0$, one more bound is required (it seems that Ricci flow prefers negative curvature here). 
Theorem 4'. For given $n \geq 3, \Lambda>0$, and $C>0$ there is an $\varepsilon>0$ with the following property. If a closed Riemannian manifold $(M, g)$ of dimension $n$ satisfies $d^{2}\|R m\|_{C^{0}} \leq \Lambda, f R^{2} \leq C \bar{R}^{2}, f|\stackrel{\circ}{R} m|^{2} \leq \varepsilon \bar{R}^{4} /\|R m\|_{C^{0}}^{2}$ or $\leq \varepsilon d^{4} \bar{R}^{4}$, and $\bar{R}>0$, then it can be deformed to a spherical space form through the Ricci flow.

The above pinching results are based upon Theorem 1 . We rely on the fact that curvature pinching implies stability. The case $\bar{R}>0$ of Theorem 3 can also be derived from the aforementioned works of Huisken, Magerin, and Nishikawa and Moser's weak maximum principle, but the case $\bar{R}<0$ is very different. In this case, crucial evolution equations used in [Hu, Ma, Ni] contain terms with the "wrong" sign and hence (also for other reasons) the approach of Huisken et al. does not work. We would also like to mention that Min-Oo and Ruh [Mi-Ru] have shown that $L^{2}$-global pinching forces the underlying manifold to be diffeomorphic to a space form. They deform the metric by the Ricci flow for a short time to achieve $C^{0}$ pinching and then apply known results. Their arguments do not yield information about long time behavior of the Ricci flow. In a similar vein, Yang [Ya] proved some $L^{p}$-curvature pinching theorems with $p>n$ (without bound on $\|R m\|_{C^{0}}$ ). (Our arguments also apply to that situation and produce time-global Ricci deformation there. Compare Remark 1.) In still another development, Gao [Gao] has treated $L^{n / 2}$-curvature pinching which involves lower bound on volume or injectivity radius. There, one may even not expect uniform short time existence of the Ricci flow.

The next two theorems again deal with curvature pinching, but avoid bounds involving diameter (the volume and Euler characteristic bounds in Theorem 2 imply diameter bounds by Gromov's work [Gr]). We define the isoperimetric constant $C_{I}$ of an $n$-dimensional Riemannian manifold $(M, g)$ to be

$$
C_{I}=\sup \left\{\frac{V(\Omega)^{n-1}}{A(\partial \Omega)^{n}}: \Omega \text { is a nonempty open set in } M, V(\Omega) \leq \frac{1}{2} V(M)\right\},
$$

where $V$ means volume and $A$ means area $((n-1)$-dimensional measure).

Theorem 5. For given $n \geq 3$ and $C>0$ there exists an $\varepsilon_{0}>0$ with the following property. If a closed Riemannian manifold $(M, g)$ of dimension $n$ satisfies $-1 \geq K^{+} \geq K^{-} \geq-1-\varepsilon \min (1,1 / \sqrt{V})$ with $0<\varepsilon \leq \varepsilon_{0}$ and $C_{I} \leq C$, then it can be deformed through the Ricci flow to an Einstein manifold with $-1+\delta(\varepsilon) \geq K^{+} \geq K^{-} \geq-1-\delta(\varepsilon)$, where $0<\delta(\varepsilon)<\frac{1}{4}$ and $\lim _{\varepsilon \rightarrow 0} \delta(\varepsilon)=0$. In dimension $n=3$, it follows that $M$ is diffeomorphic to a hyperbolic space form.

Remark 4. One should note that the conditions in this theorem are weaker than those in Theorem 2, because $C_{I}$ can be estimated in terms of Ricci curvature, diameter and volume. It is unknown whether (for $n \geq 4$ ) Einstein manifolds of negative and sufficiently pinched curvature must be isometrically or at least topologically space forms. (The answer for positive curvature is positive, see $\S 2$.) On the other hand, the difference between the assumptions of these two theorems is somewhat subtle. In Theorem 5 the dependence of pinching on volume is explicitly on the order of $1 / \sqrt{V}$, while in Theorem 2 this dependence is inexplicit and probably on a much higher order. Our interest in Theorem 5 (for 
$n \neq 3$ ) is the hope to use it to produce negatively pinched but diffeomorphically nonhyperbolic Einstein manifolds. So far we have not been able to do so. The only known examples of diffeomorphically nonhyperbolic manifolds with almost zero pinched negative curvature due to Gromov-Thurston [Gr-Th] and Farrell-Jones [Fa-Jo] do not satisfy the condition of Theorem 5. The pinching there is on the order of $1 / \log V$.

The $L^{2}$-version of Theorem 5 involves curvature bounds normalized by volume and yields stronger information, namely deformation to space forms in all dimensions:

Theorem 6. For given $n \geq 3, \Lambda>0$, and $C>0$ there exists an $\varepsilon>0$ such that the following is true. If a closed Riemannian manifold $(M, g)$ of dimension $n$ satisfies $V^{2 / n}\|R m\|_{C^{0}} \leq \Lambda, C_{I} \leq C, \bar{R}<0$, and $f|\stackrel{R}{R} m|^{2} \leq \varepsilon \bar{R}^{4} /\|R m\|_{C^{0}}^{2}$ (or $\left.\leq \varepsilon d^{4} \bar{R}^{4}\right)$, then it can be deformed to a hyperbolic space form through the Ricci flow. In particular, $M$ is diffeomorphic to a hyperbolic space form.

Remark 5. In dimensions $n \geq 4$, this theorem can also be thought of as an $L^{2}$-version of Theorem 2 . Finally we notice that in Theorem 1 one can also adopt a bound on the isoperimetric constant in order to avoid diameter.

The arguments in this paper also apply to many other geometric flow problems such as the mean curvature flow and the heat flow for harmonic mappings.

It is a pleasure to thank Professor R. Schoen for his interest in this work.

\section{RICCI PINCHING}

Contrary to the approach in [Ha1], where one first considers the unnormalized Ricci flow $\partial g / \partial t=-2 R c$, we work directly with the (normalized) Ricci flow $(0.2)$. We first derive evolution equations for curvatures. The dot denotes $\partial / \partial t$ when it is on top or a superscript, while the dot in the middle denotes inner product. Let $R_{i j}, T_{i j}$ be the coefficients of $R c, T$ and $B_{i j k l}=R^{p}{ }_{j}{ }_{j} R_{p k q l}$. We fix a closed manifold $M$ of dimension $n \geq 3$.

Lemma 1. Along the Ricci flow we have

$$
\begin{gathered}
\dot{R}_{i j k l}=\Delta R_{i j k l}+2\left(B_{i j k l}-B_{i j l k}-B_{i l j k}+B_{i k j l}\right) \\
-R_{p j k l} R_{i}^{p}-R_{i p k l} R_{j}^{p}-R_{i j p l} R_{k}^{p}-R_{i j k p} R_{l}^{p}+\frac{2 \bar{R}}{n} R_{i j k l}, \\
\dot{R}_{i j}=\Delta R_{i j}+2 R_{i p j q} R^{p q}-2 R_{i p} R_{j}^{p}, \\
\dot{T}_{i j}=\Delta T_{i j}+2 R_{i p j q} T^{p q}-\frac{2|T|^{2}}{n} g_{i j}-2 T_{p i} T^{p} j, \\
\dot{R}=\Delta R+2|T|^{2}+\frac{2 \delta R}{n} R \\
\frac{\partial}{\partial t}|T|^{2}=\Delta|T|^{2}-2|\nabla T|^{2}+4 R m(T) \cdot T+\frac{4}{n} \delta R|T|^{2}, \\
\frac{\partial}{\partial t} \int|T|^{2}=-2 \int|\nabla T|^{2}+4 \int R m(T) \cdot T=\left(1-\frac{4}{n}\right) \int \delta R|T|^{2} .
\end{gathered}
$$


Proof. (1) follows from the calculations in Hamilton's paper [Ha1]. The other identities follow straightforwardly from (1) and (0.2).

For the convenience of the reader, we summarize some basic facts.

Lemma 2 (short time existence). The Ricci flow exists (and is smooth) on the time interval $[0, \tau(n) / \wedge]$, where $\wedge$ denotes $\|R m\|_{C^{0}}$ at $t=0$ and $\tau(n)$ is a positive constant depending only on $n$. Moreover, the following estimates hold at any time $t \in(0, \tau(n) / \wedge]$ :

$$
\left\|\nabla^{k} R m\right\|_{C^{0}} \leq \frac{c(k, n)}{t^{k / 2}} \wedge, \quad k=0,1, \ldots,
$$

where $c(k, n)$ depends only on $k$ and $n$. We can actually choose $c(0, n)=2$.

This follows from Theorem 14.1 in [Hal] and the maximum principle; see [Ha3] or [Sh].

For a given metric $g$ on $M$ and $A \geq 1$, the Sobolev $A$-constant $C_{S}^{(A)}$ of $g$ (or better: of the Riemannian manifold $(M, g)$ ) is defined to be the smallest positive number for which the following Sobolev inequality holds:

$$
\left(\int|f|^{2 n /(n-2)}\right)^{(n-2) / n} \leq C_{S}^{(A)} \int|\nabla f|^{2}+A V^{-2 / n} \int f^{2}, \quad f \in C^{\infty}(M) .
$$

We simply call $C_{S}^{(1)}$ "Sobolev constant" and denote it by $C_{S} \quad\left(C_{S}\right.$ does exist; see $[\mathrm{Li}])$.

Lemma 3 (Moser's weak maximum principle). Let $g=g(t), 0 \leq t \leq T$, be a smooth family of metrics, $b$ a nonnegative constant, and $f$ a nonnegative function on $M \times[0, T]$ which satisfies the partial differential inequality

$$
\partial f / \partial t \leq \Delta f+b f \text { on } M \times[0, T],
$$

where $\Delta$ refers to $g(t)$. Then for any $x \in M, t \in(0, T]$,

$$
|f(x, t)| \leq c\left(b+l+\frac{1}{t}\right)^{1 / 2}\left(C_{S}^{(A)}\left(b+l+\frac{1}{t}\right)+A V^{-2 / n}\right)^{n / 4} e^{c b t}\left\|f_{0}\right\|_{L^{2}},
$$

for arbitrary $A \geq 1$,

$$
|f(x, t)| \leq c \frac{1}{\sqrt{V}} e^{c H d} \max \left(1, d^{n / 2}\right)\left(b+l+1+\frac{1}{t}\right)^{(1+n / 2) / 2} e^{c b t}\left\|f_{0}\right\|_{L_{2}},
$$

where $c$ is a positive constant depending only on $n$,

$$
\begin{aligned}
& \quad l=\max _{0 \leq t \leq T}\left|\frac{d}{d t}(d v) / d v\right|, \quad C_{S}^{(A)}=\max _{0 \leq t \leq T} C_{S}^{(A)}(g(t)), \quad V=\min _{0 \leq t \leq T} V(g(t)), \\
& \quad d=\max _{0 \leq t \leq T} \operatorname{diam} g(t), \quad H=\max _{0 \leq t \leq T} \sqrt{\|R\|_{C^{0}}}, \quad \text { and } f_{0}=f(\cdot, 0) \\
& \text { (diam }=\text { diameter). }
\end{aligned}
$$

This lemma follows from (a simple modification of) the proof of Theorem 4.1 in [Ya] and the estimates for the Sobolev constant given in [Gal]. See also the original paper [Mo] of Moser.

Next we study how stability evolves along the Ricci flow. For each presentation let us first introduce a definition. 
Definition 3. Let $g$ be a metric. For any positive number $\beta$ we define the $\beta$-value $\lambda_{\beta}$ of $g$ to be

$$
\begin{aligned}
& \lambda_{\beta}=\inf \left\{Q(h) / \int|h|^{2}: h\right. \text { is a traceless symmetric 2-tensor } \\
& \text { with } \left.\|h\|_{C^{0}}^{2} \leq \frac{1}{\beta} \int|h|^{2}\right\} .
\end{aligned}
$$

Lemma 4. Assume that the initial metric $g_{0}$ of the Ricci flow satisfies $\lambda_{e}>0$ and $\|R m\|_{C^{0}} \leq 1$. Set $\beta_{0}=\int_{M_{0}}|T|^{2}$, where $M_{t}$ means $M$ equipped with the metric $g_{t}$ at time $t$. Then there are numbers $0<a(n)<\min (1, \tau(n)), c(n)>1$ depending only on $n$ such that for $0 \leq t \leq a(n) \lambda_{e}^{2} /\left(1+\lambda_{e}^{2}\right)$, the $\beta_{0}$-value $\lambda_{\beta_{0}}$ of $g=g_{t}$ satisfies $\lambda_{\beta_{0}} \geq \lambda_{e} / c(n)\left(1+\lambda_{e}\right) .(\tau(n)$ was introduced in Lemma 2 .)

Proof. Consider the Einstein form $Q$ along the flow. Fix a symmetric 2-tensor $h$. We put $Q=Q(h)$ and compute

$$
\begin{aligned}
\dot{Q}= & 2 \int \dot{\nabla} h \cdot \nabla h+\int\langle\nabla h, \nabla h\rangle+\int\left(|\nabla h|^{2}-2 R m(h) \cdot h+T(h) \cdot h\right)(d v)^{\bullet} \\
& -2 \int(R m(h) \cdot h)^{\bullet}+\int(T(h) \cdot h)^{\bullet}
\end{aligned}
$$

where \langle\rangle denotes the inner product relative to $g(t)$. From the Ricci flow equation (0.2) one easily deduces

$$
\begin{gathered}
(d v)^{\cdot}=-\delta R d v, \\
|\langle\nabla h, \nabla h\rangle| \leq C_{1}(|T|+|\delta R|)|\nabla h|^{2} .
\end{gathered}
$$

Here and in the sequel, $C_{1}, C_{2}, \ldots$ denote various positive constants depending only on $n$. From [Be, 1.174] and (0.2) we obtain $|\dot{\nabla} h| \leq C_{2}|\nabla R c||h|$. But the contracted second Bianchi identity implies (note $n \geq 3$ )

$$
|\nabla R c| \leq C_{3}|\nabla T|
$$

Hence we have

$$
|\dot{\nabla} h| \leq C_{4}|\nabla T||h| \text {. }
$$

Next we derive from [Be, 1.174] and (0.2)

$$
\dot{R} m=l\left(\nabla^{2} R c\right)+q\left(R m, T, g^{-1}\right)+q_{0}(R m, \delta R),
$$

where $l, q, q_{0}$ denote some (universal) linear forms and $g^{-1}$ is the tensor $g^{i j}$. This combined with integration by parts and (1.4) (as well as simple computation of $\dot{\langle}$,$\left.\rangle and \partial g^{-1} / \partial t\right)$ yields

$$
\left|\int(R m(h) \cdot h)^{\cdot}\right| \leq C_{5} \int\left(|\nabla T||\nabla h||h|+|R m||T||h|^{2}+|R m||\delta R||h|^{2}\right) .
$$

Finally, we apply equation (3) in Lemma 1 and integration by parts to deduce (1.8) $\left|\int(T(h) \cdot h)^{\cdot}\right| \leq C_{6} \int\left(|T|^{2}+|T||\delta R|+|R m||T|\right)|h|^{2}+C_{6} \int|\nabla T||\nabla h||h|$. 
These estimates together with Lemma 2 and the condition $\|R m\|_{C^{0}}=1$ at $t=0$ imply for $0 \leq t \leq \tau(n)$

$$
\begin{aligned}
|\dot{Q}| & \leq C_{7} \int\left(|\nabla T||\nabla h||h|+|\nabla h|^{2}+|h|^{2}\right) \\
& \leq C_{7}\left(1+\frac{1}{\varepsilon}\right) \int|\nabla h|^{2}+C_{7} \varepsilon \int|\nabla T|^{2}|h|^{2}+C_{7} \int|h|^{2} \\
& \leq C_{7}\left(1+\frac{1}{\varepsilon}\right) Q+C_{8} \varepsilon \int|\nabla T|^{2}|h|^{2}+C_{8}\left(1+\frac{1}{\varepsilon}\right) \int|h|^{2},
\end{aligned}
$$

where $\varepsilon>0$ is to be chosen. Integration yields

$$
Q \geq Q_{0} e^{-C_{7}(1+1 / \varepsilon) t}-C_{8} \int_{0}^{t}\left(\varepsilon \int_{M_{s}}|\nabla T|^{2}|h|^{2}+\left(1+\frac{1}{\varepsilon}\right) \int_{M_{s}}|h|^{2}\right) d s
$$

where $Q_{t}$ means $Q$ at time $t$. Now we integrate equation (6) in Lemma 1 to obtain

$$
\int_{0}^{t} d s \int_{M_{s}}|\nabla T|^{2} \leq \frac{1}{2} \int_{M_{0}}|T|^{2}-\frac{1}{2} \int_{M_{t}}|T|^{2}+C_{9} \int_{0}^{t} d s \int_{M_{s}}|T|^{2} .
$$

Writing $\varphi(t)=\int_{0}^{t} d s \int_{M}|T|^{2}$ we see from (1.11) that

$$
\dot{\varphi} \leq \int_{M_{0}}|T|^{2}+2 C_{9} \varphi
$$

whence

$$
\varphi(t) \leq \frac{1}{2 C_{9}} e^{2 C_{9} t} \int_{M_{0}}|T|^{2}
$$

We conclude

$$
\int_{0}^{t} d s \int_{M_{s}}|\nabla T|^{2} \leq C_{10} \int_{M_{0}}|T|^{2} \quad(t \leq \tau(n)) .
$$

To proceed we observe that by Lemma 2 and the flow equation (0.2), we have the following comparison between the metrics $g=g_{t}, 0 \leq t \leq \tau(n)$,

$$
\frac{1}{C_{11}} g_{0} \leq g \leq C_{11} g_{0}, \quad\left\|g-g_{0}\right\|_{C^{0}\left(M_{0}\right)} \leq C_{12} t .
$$

Now we fix a time $\tau \leq \tau(n)$ and assume that $h$ is traceless relative to $g_{\tau}$ and satisfies

$$
\|h\|_{C^{0}\left(M_{\tau}\right)}^{2} \leq \frac{1}{\beta_{0}} \int_{M_{\tau}}|h|^{2}
$$

We set

$$
h^{0}=h-\frac{\operatorname{tr}_{g_{0}} h}{n} g_{0}
$$

and replace $Q(h)$ by $Q\left(h^{0}\right)$ in the previous estimates. From (1.10), (1.12), (1.14), and (1.13) we derive

$$
Q_{\tau}\left(h^{0}\right) \geq Q_{0}\left(h^{0}\right) e^{-C_{7}(1+1 / \varepsilon) \tau}-\varepsilon C_{13} \int_{M_{0}}|h|^{2}-C_{8}\left(1+\frac{1}{\varepsilon}\right) \tau \int_{M_{0}}\left|h^{0}\right|^{2} .
$$


But $\left|\operatorname{tr}_{g_{0}} h\right|=\left|\operatorname{tr}_{g_{0}} h-\operatorname{tr}_{g_{\tau}} h\right| \leq C_{14}|h|_{g_{0}} \tau$ by (1.13). Hence

$$
\left|h-h^{0}\right|_{g_{0}} \leq C_{15} \tau|h|_{g_{0}} \text { and } \int_{M_{0}}|h|^{2} \leq 4 \int_{M_{0}}\left|h^{0}\right|^{2}
$$

provided that $\tau \leq \tau_{0}(n):=1 / 2 C_{15}$ (for the second inequality). Choosing a suitable $\varepsilon$ in (1.15) we then get

$$
\begin{aligned}
Q_{\tau}\left(h^{0}\right) \geq & \frac{1}{2} Q_{0}\left(h^{0}\right) \geq \frac{1}{2}(1-a) \int_{M_{0}}\left|\nabla h^{0}\right|^{2} \\
& -\frac{1}{2}(1-a) C_{16} \int_{M_{0}}\left|h^{0}\right|^{2}+\frac{1}{2} a \lambda_{e} \int_{M_{0}}\left|h^{0}\right|^{2}
\end{aligned}
$$

for $a \in(0,1)$, provided that $\tau \leq \tau_{1}\left(n, \lambda_{e}\right):=\min \left\{\lambda_{e}, \lambda_{e}^{2}\right\} / C_{17}$ for some $C_{17}$. Choosing $a=C_{16} /\left(C_{16}+\lambda_{e} / 2\right)$ we conclude (we may assume $C_{16}>1$ )

$$
Q_{\tau}\left(h^{0}\right) \geq \frac{1}{4} \frac{\lambda_{e}}{C_{16}+\lambda_{e} / 2} \int_{M_{0}}\left(\left|\nabla h^{0}\right|^{2}+\left|h^{0}\right|^{2}\right) .
$$

Our purpose is however to estimate $Q_{\tau}(h)$. We have

$$
\left|Q_{\tau}(h)-Q_{\tau}\left(h^{0}\right)\right| \leq 2 \int_{M_{\tau}}\left|\nabla\left(h-h^{0}\right)\right||\nabla h|+2 C_{18} \int_{M_{\tau}}\left|h-h^{0}\right||h| .
$$

We estimate (at time $\tau$ )

$$
\begin{aligned}
\left|\nabla\left(h-h^{0}\right)\right| & \leq \frac{\left|\operatorname{tr}_{g_{0}} h\right|}{n}\left|\nabla g_{0}\right|+\frac{\left|g_{0}\right|}{n}\left|\nabla \operatorname{tr}_{g_{0}} h\right| \\
& \leq C_{19}\left(\left|\nabla_{g_{0}}\right|\left|h^{0}\right|+\tau|\nabla h|\right),
\end{aligned}
$$

because of $(1.16),(1.13)$, and since

$$
\left|\nabla \operatorname{tr}_{g_{0}} h\right|=\left|\nabla\left(\left(g_{0}^{i j}-g^{i j}\right) h_{i j}\right)\right| \leq C_{20}\left(\left|\nabla g_{0}\right||h|+\tau|\nabla h|\right),
$$

which follows from (1.13). But we have from (1.5) and (1.13)

$$
\left|\nabla g_{0}\right| \leq C_{21} \int_{0}^{\tau}|\nabla T| d t, \quad\left|\nabla h^{0}\right| \leq\left|\nabla h^{0}\right|_{t=0}+C_{22}\left|h^{0}\right| \int_{0}^{\tau}|\nabla T| d t .
$$

Hence

$$
|\nabla h| \leq\left|\nabla h^{0}\right|_{t=0}+C_{23}\left|h^{0}\right| \int_{0}^{\tau}|\nabla T| d t,
$$

provided that $\tau \leq \tau_{2}(n):=1 / 2 C_{19}$.

Now we deduce from (1.19), (1.20), (1.23), (1.16), and (1.13)

$$
\begin{aligned}
\left|Q_{\tau}(h)-Q_{\tau}\left(h^{0}\right)\right| \leq C_{24}\left(\tau \int_{M_{0}}\left|\nabla h^{0}\right|^{2}+\int_{M_{0}}\left|h^{0}\right|\left|\nabla h^{0}\right| \int_{0}^{\tau}|\nabla T| d t\right. \\
\left.\quad+\int_{M_{0}}\left|h^{0}\right|^{2}\left(\int_{0}^{\tau}|\nabla T| d t\right)^{2}+\tau \int_{M_{0}}\left|h^{0}\right|^{2}\right) .
\end{aligned}
$$

Here everything is measured in $g_{0}$ except in the integral $\int_{0}^{\tau}|\nabla T| d t$, in which $|\nabla T|$ is measured in $g_{t}, 0 \leq t \leq \tau$. But we observe by (1.13) that

$$
\int_{M_{0}} \int_{0}^{\tau}|\nabla T|^{2} d t \leq C_{25} \int_{0}^{\tau} d t \int_{M_{t}}|\nabla T|^{2} .
$$


Hence by the Hölder inequality

(1.24)

$$
\begin{aligned}
& \left|Q_{\tau}(h)-Q_{\tau}\left(h^{0}\right)\right| \\
& \quad \leq C_{26}(\tau+\sqrt{\tau})\left(\int_{M_{0}}\left|\nabla h^{0}\right|^{2}+\left\|h^{0}\right\|_{C^{0}\left(M_{0}\right)}^{2} \int_{0}^{\tau} d t \int_{M_{t}}|\nabla T|^{2}+\int_{M_{0}}\left|h^{0}\right|^{2}\right) .
\end{aligned}
$$

We can apply $(1.14),(1.12)$, and (1.13) to (1.24). Then we derive (recall $\beta_{0}=$ $\left.\int_{M_{0}}|T|^{2}\right)$

$$
\left|Q_{\tau}(h)-Q_{\tau}\left(h^{0}\right)\right| \leq C_{27}(\tau+\sqrt{\tau}) \int_{M_{0}}\left(\left|h^{0}\right|^{2}+\left|\nabla h^{0}\right|^{2}\right) .
$$

On account of (1.18), (1.25), and (1.13) we finally arrive at

$$
Q_{\tau}(h) \geq \frac{\lambda_{3}}{C_{28}\left(C_{28}+\lambda_{e}\right)} \int_{M_{\tau}}|h|^{2}
$$

for some $C_{28}$, provided that $\tau \leq \min \left\{\tau(n), \tau_{0}(n), \tau_{1}\left(n, \lambda_{e}\right), \tau_{2}(n), \tau_{3}\left(n, \lambda_{e}\right)\right\}$, where $\tau_{3}\left(n, \lambda_{e}\right)$ satisfies the equation $C_{27}(t+\sqrt{t})=\frac{1}{8} \frac{\lambda_{e}}{C_{16}+\lambda_{e} / 2}$.

We expect that the $\beta_{0}$-value or $\beta$-value for suitable $\beta$ of $g_{t}$ continues to be bounded away from zero for long time. Our argument is to prove this along with the convergence of the Ricci flow. The idea is that $L_{2}$-decay of the traceless Ricci tensor $T$ and hence the convergence of the flow will follow from a positive lower bound for $\lambda_{\beta}$. On the other hand, the decay of $T$ helps us to establish such a bound. This sounds like a circle, but we have enough gap to play with.

We continue under the assumption of Lemma 4. Set

$$
\tau_{0}=\frac{1}{2} a(n) \frac{\lambda_{e}^{2}}{1+\lambda_{e}^{2}}, \quad \gamma_{0}=\frac{\lambda_{e}}{c(n)\left(1+\lambda_{e}\right)} .
$$

We say that a (finite) time $\tau \geq \tau_{0}$ satisfies Condition $\mathbf{B}$, for $B=\left(b_{0}, b_{1}, \gamma\right)$ satisfying $b_{0} \geq 1, b_{1}>0$, and $0<\gamma \leq \gamma_{0} / 4$, if the following is true:

(i) $\lambda_{\beta}>\gamma_{0} / 2$ on $[0, \tau]$, with $\beta=4 \int_{M_{0}}|T|^{2}$,

(ii) $\|R m\|_{C^{0}}<10$ on $[0, \tau]$,

(iii) $\int_{M_{t}}|T|^{2}<b_{0} e^{-\gamma t} \int_{M_{0}}|T|^{2}$ for $t \in[0, \tau]$,

(iv) $\operatorname{diam}\left(M_{t}\right)<b_{1} d_{0}$ for $t \in[0, \tau]$, where $d_{0}=\operatorname{diam} M_{0}$.

Assuming $\tau$ satisfies Condition B for some $B$, we are going to check whether the estimates assumed in this condition can be used to produce better ones. The ultimate purpose is to employ suitable estimates to show that Condition B is an open as well as closed condition. First note, on account of Lemma 2 (we are tired of the $C_{k}$ 's, so we change to $\left.a_{k}=a_{k}(n)\right)$

$$
\|\nabla R m\|_{C^{0}} \leq a_{1} / \sqrt{\tau_{0}}, \quad\left\|\nabla^{2} R m\right\|_{C^{0}} \leq a_{2} / \tau_{0}, \quad\left\|\nabla^{3} R m\right\|_{C^{0}} \leq a_{3} / \sqrt[3]{\tau^{2}}
$$

on $\left[\tau_{0} / 4, \tau\right]$. We put

$$
\varepsilon=f_{M_{0}}|T|^{2}, \quad V_{0}=V\left(g_{0}\right)
$$

To estimate $\|T\|_{C^{0}}$, we derive from equation (5) in Lemma 1 and (ii) above

$$
\partial|T| / \partial t \leq \Delta|T|+a_{4}|T|
$$


on $[0, \tau]$ (in the weak sense). Applying Lemma 3 to this differential inequality, we derive

$$
\|T\|_{C^{0}\left(M_{t}\right)}^{2} \leq \frac{a_{5}}{\tau_{0}^{n}} e^{a_{5} b_{1} d_{0}} \int_{M_{t-\tau_{0} / 4}}|T|^{2} \leq \frac{a_{5}}{\tau_{0}^{n}} e^{a_{5} b_{1} d_{0}+1} b_{0} \varepsilon e^{-\gamma t} V_{0}
$$

on $\left[\tau_{0} / 4, \tau\right]$. To estimate $\|\nabla T\|_{C^{0}}$ we first apply the interpolation inequality 12.7 in [Ha1] together with (iii) and (1.27) to get

$$
\int_{M_{t}}|\nabla T|^{2} \leq \frac{a_{6}}{\tau_{0}} \sqrt{b_{0} \varepsilon} e^{-\gamma t / 2} V_{0}, \quad t \in\left[\tau_{0} / 4, \tau\right] .
$$

Next we observe the following evolution equation:

$$
\begin{aligned}
\frac{\partial}{\partial t}|\nabla T|^{2}= & \Delta|\nabla T|^{2}-2\left|\nabla^{2} T\right|^{2}+R m * \nabla T * \nabla T \\
& +\nabla R m * T * \nabla T+\delta R(\nabla T * \nabla T),
\end{aligned}
$$

where $*$ means various linear combinations (involving contraction). This equation can be derived from equation (3) in Lemma 1 with the help of Lemma 13.1 in [Ha1]. Combining (1.30) with (1.27), (1.28), and (ii) yields for $t \in\left[\tau_{0} / 4, \tau\right]$

$$
\frac{\partial}{\partial t}|\nabla T| \leq \Delta|\nabla T|+a_{7}|\nabla T|+\frac{a_{7}}{\tau_{0}^{n / 2}} e^{a_{7} b_{1} d_{0}} \sqrt{b_{0} \varepsilon} e^{-\gamma t / 2} \sqrt{V_{0}}
$$

Hence, by virtue of Lemma 3 and (1.29),

$$
\begin{aligned}
\|\nabla T\|_{C^{0}\left(M_{t}\right)} & \leq \frac{a_{8}}{\sqrt{V_{0}}} e^{a_{8} b_{1} d_{0}} \tau_{0}^{-(1+n / 2) / 2}\left(\sqrt{\frac{V_{0}}{\tau_{0}}} \sqrt[4]{b_{0} \varepsilon} e^{-\gamma t / 4}+\frac{\sqrt{b_{0} \varepsilon V_{0}}}{\gamma \tau_{0}^{(n+1) / 2}} e^{-\gamma t / 2}\right) \sqrt{V_{0}} \\
& \leq \frac{2 a_{8}}{\gamma \tau_{0}^{n+1}} e^{a_{8} b_{1} d_{0}} b_{0} \sqrt[4]{\varepsilon} e^{-\gamma t / 4} \sqrt{V_{0}}, \quad t \in\left[\tau_{0} / 2, \tau\right] .
\end{aligned}
$$

On account of (1.4) we immediately conclude

$$
\|\nabla R c\|_{C^{0}\left(M_{t}\right)}+\|\delta R\|_{C^{0}\left(M_{t}\right)} \leq \frac{a_{9}}{\gamma \tau_{0}^{n+1}} e^{a_{9} b_{1} d_{0}} b_{0} \sqrt[4]{\varepsilon} e^{-\gamma t / 4} \sqrt{V_{0}}, \quad t \in\left[\tau_{0} / 2, \tau\right] .
$$

The second order derivative $\nabla^{2} T$ can be treated in a similar way. Thus, the interpolation inequality quoted before produces for $t \in\left[\tau_{0} / 4, \tau\right]$

$$
\int_{M_{t}}\left|\nabla^{2} T\right|^{2} \leq \frac{a_{10}}{\sqrt{\tau_{0}}} \sqrt[3]{b_{0} \varepsilon} e^{-\gamma t / 3} V_{0}
$$

From an evolution equation for $\left|\nabla^{2} T\right|^{2}$ similar to (1.30) one then derives for $t \in\left[\tau_{0} / 2, \tau\right]$

$$
\left\|\nabla^{2} T\right\|_{C^{0}\left(M_{t}\right)} \leq\left(a_{11} / \gamma^{2} \tau_{0}^{2 n}\right) e^{a_{11} b_{1} d_{0}} b_{0} \sqrt[6]{\varepsilon} e^{-\gamma t / 6} \sqrt{V_{0}}
$$

Next we estimate $\nabla^{2} R$. First we derive from equation (4) in Lemma 1 the following evolution equation:

$$
\begin{aligned}
\frac{\partial}{\partial t}\left|\nabla^{2} R\right|^{2}= & \Delta\left|\nabla^{2} R\right|^{2}-2\left|\nabla^{3} R\right|^{2}+R m * \nabla^{2} R * \nabla^{2} R \\
& +\nabla R m * \nabla R * \nabla^{2} R+\nabla T * \nabla T * \nabla^{2} R \\
& +\nabla^{2} T * T * \nabla^{2} R+\delta R\left(\nabla^{2} R * \nabla^{2} R\right)+R\left(\nabla^{2} T * \nabla^{2} R\right)
\end{aligned}
$$


Using this equation, the above proven estimates as well as an interpolation estimate for $\int\left|\nabla^{2} R\right|^{2}$ we then deduce for $t \in\left[\tau_{0}, \tau\right]$

$$
\left\|\nabla^{2} R\right\|_{C^{0}\left(M_{t}\right)} \leq\left(a_{12} / \gamma^{3} \tau_{0}^{3 n}\right) e^{a_{12} b_{1} d_{0}} b_{0} \sqrt[8]{\varepsilon} e^{-\gamma t / 8} \sqrt{V_{0}} .
$$

Estimate of $R m$. By Lemma 2, equation (1.6), and estimates (1.33), (1.34), and (1.35), we have on $\left[\tau_{0}, \tau\right]$

$$
\frac{\partial}{\partial t}|R m|^{2} \leq a_{13}(|\dot{R} m|+|T|+|\delta R|) \leq \frac{a_{14}}{\gamma^{3} \tau_{0}^{3 n}} e^{a_{14} b_{1} d_{0}} b_{0} \sqrt[8]{\varepsilon} e^{-\gamma t / 8} \sqrt{V_{0}},
$$

hence

$$
\|R m\|_{C^{0}\left(M_{\tau}\right)}^{2} \leq 4+\left(8 a_{14} / \gamma^{4} \tau_{0}^{3 n}\right) e^{a_{14} b_{1} d_{0}} b_{0} \sqrt[8]{\varepsilon} \sqrt{V_{0}} .
$$

Estimate of diam. For a fixed tangent vector $v$ we compute

$$
\frac{\partial}{\partial t}|v|^{2}=\dot{g}(v, v)=-2 T(v, v)-\frac{2 \delta R}{n}|v|^{2},
$$

whence, by the previous estimates,

$$
\left.\left.\left|\frac{\partial}{\partial t}\right| v\right|^{2}\left|\leq \frac{a_{15}}{\gamma \tau_{0}^{n+1}} e^{a_{15} b_{3} d_{0}} b_{0} \sqrt[4]{\varepsilon} e^{-\gamma t / 4} \sqrt{V_{0}}\right| v\right|^{2}
$$

on $\left[\tau_{0}, \tau\right]$. We derive for $t \in\left[\tau_{0}, \tau\right]$

$$
e^{-A} g_{\tau_{0}} \leq g_{t} \leq e^{A} g_{\tau_{0}} \quad \text { with } A=\left(4 a_{15} / \gamma^{2} \tau_{0}^{n+1}\right) e^{a_{15} b_{3} d_{0}} b_{0} \sqrt[4]{\varepsilon} \sqrt{V_{0}}
$$

Together with (1.13) this shows

$$
\operatorname{diam} M_{\tau} \leq \sqrt{c_{11}} e^{A / 2} d_{0} .
$$

Estimate of $\int|T|^{2}$. We make the following

Assumption I. $\|\delta R\|_{C_{0}} \leq \gamma_{0} / 2$ holds on $\left[\tau_{0}, \tau\right]$.

We say that $t \in\left[\tau_{0}, \tau\right]$ is an $e$-time, provided that

$$
\frac{1}{2} \int_{M_{t-\tau_{0} / 4}}|T|^{2} \geq e \int_{M_{t}}|T|^{2}
$$

We consider the two possible cases.

Case 1 . There is no $e$-time.

In this case we notice that from (1.28),

$$
\|T\|_{C^{0}\left(M_{t}\right)}^{2} \leq \frac{2 a_{5}}{\tau_{0}^{n}} e^{a_{5} b_{1} d_{0}+1} \varepsilon V_{0} \frac{1}{\beta_{0}} \int_{M_{t}}|T|^{2}
$$

(recall $\beta_{0}=\int_{M_{0}}|T|^{2}$ ) for $t \in\left[\tau_{0}, \tau\right]$. We make

Assumption II. $\left(a_{5} / \tau_{0}^{n}\right) e^{a_{5} b_{1} d_{0}+1} \varepsilon V_{0} \leq 1 / 8$.

By (i) we then derive

$$
Q(T)>\frac{\gamma_{0}}{2} \int|T|^{2} \text { on }\left[\tau_{0}, \tau\right] .
$$

This estimate, Assumption I, and equation (6) in Lemma 1 imply

$$
\int_{M_{\tau}}|T|^{2} \leq e^{-\gamma_{0}\left(\tau-\tau_{0}\right) / 2} \int_{M_{\tau_{0}}}|T|^{2} \leq e^{-\tau_{0} \gamma_{0} / 4} b_{0} e^{-\gamma \tau} \int_{M_{0}}|T|^{2} .
$$


Here condition (iii) and the assumption $\gamma \leq \gamma_{0} / 4$ have been used.

Case 2. There are e-times.

Let $\tau_{1}$ be the maximum $e$-time. We apply the above argument to the interval $\left[\tau_{1}, \tau\right]$ and deduce

$$
\int_{M_{\tau}}|T|^{2} \leq e^{-\gamma_{0}\left(\tau-\tau_{1}\right) / 2} \int_{M_{\tau_{1}}}|T|^{2} \leq \frac{1}{2 e} e^{-\gamma_{0}\left(\tau-\tau_{1}\right) / 2} \int_{M_{\tau_{1}-\tau_{0}}}|T|^{2} \leq \frac{1}{2} b_{0} e^{-\gamma \tau} \int_{M_{0}}|T|^{2} .
$$

We conclude in both cases

$$
\int_{M_{\tau}}|T|^{2} \leq A_{1} b_{0} e^{-\gamma \tau} \int_{M_{0}}|T|^{2}
$$

with $A_{1}=\max \left(\frac{1}{2}, e^{-\tau_{0} \gamma_{0} / 4}\right)$.

Estimate of $\lambda_{\beta}$. We compare the Einstein form of $g_{\tau}$ with that of $g_{\tau_{0}}$ and follow the arguments in the proof of Lemma 4 . But here we have to appeal to the decay estimates assumed in and derived from Condition B, because $\tau$ can be large. We choose $\beta=2 \int_{M_{0}}|T|^{2}=2 \beta_{0}$.

From (1.1), (1.2), (1.3), (1.5), (1.7), (1.8) and the above estimates for $R m$, $\nabla T, \delta R$ we deduce on $\left[\tau_{0}, \tau\right]$ for $Q=Q(h), h$ being a fixed symmetric 2-tensor,

$$
\begin{aligned}
|\dot{Q}| & \leq a_{16} \int\left(|\nabla T||\nabla h||h|+(|T|+|\delta R|)|\nabla h|^{2}+(|T|+|\delta R|)\left(|h|^{2}\right)\right. \\
& \leq \frac{a_{17}}{\gamma \tau_{0}^{n+1}} e^{a_{17} b_{1} d_{0}} b_{0} \sqrt[4]{\varepsilon} e^{-\gamma t / 4} \sqrt{V_{0}} \int\left(|\nabla h|^{2}+|h|^{2}\right) \\
& \leq \frac{1}{\gamma \tau_{0}^{n+1}} e^{a_{17} b_{1} d_{0}} b_{0} \sqrt[4]{\varepsilon} e^{-\gamma t / 4} \sqrt{V_{0}}\left(a_{17} Q+a_{18} \int|h|^{2}\right) .
\end{aligned}
$$

We restrict to those $h$ which satisfy

$$
Q_{\tau_{0}}(h) \geq \frac{4 \gamma_{0}}{5} \int_{M_{\tau_{0}}}|h|^{2}
$$

Claim. Then we have on $\left[\tau_{0}, \tau\right]$

$$
Q \geq \frac{2 \gamma_{0}}{3} \int_{M_{t}}|h|^{2}
$$

provided that the following is satisfied.

Assumption III. $A_{2} e^{-A_{3}} \geq \frac{3}{4}$, where $A_{2}, A_{3}$ will be defined below.

In fact, if we put $\Gamma=\left\{t \in\left[\tau_{0} \tau\right]:(1.42)\right.$ holds $\}$, then $\Gamma$ is closed. We show that it is also open in $\left[\tau_{0}, \tau\right]$. For $t_{0} \in \Gamma$ we have from $(1.40),(1.41)$

$$
Q_{t_{0}} \geq A_{2} Q_{\tau_{0}} \geq \gamma_{0} A_{2} \int_{M_{\tau_{0}}}|h|^{2}
$$

with

$$
A_{2}=\frac{4}{5} \exp \left(\frac{-4 \sqrt{V_{0}}}{\gamma^{2} \tau_{0}^{n+1}} e^{a_{17} b_{1} d_{0}} b_{0} \sqrt[4]{\varepsilon}\left(a_{17}+\frac{3 a_{18}}{2 \gamma_{0}}\right)\right)
$$


We can compare $\int_{M_{\tau_{0}}}|h|^{2}$ with $\int_{M_{t_{0}}}|h|^{2}$ by means of (1.37), but a more convenient way is to compute

$$
\frac{\partial}{\partial t}|h|^{2}=4\left(T^{i k}+\frac{\delta R}{n} g^{i k}\right) h_{i}^{l} h_{k l}
$$

thus (using (1.2))

$$
\frac{\partial}{\partial t} \int_{M_{t}}|h|^{2}=4 \int_{M_{t}}\left(T^{i k}+\frac{\delta R}{n} g^{i k}\right) h_{i}{ }^{l} h_{k l}-\frac{1}{2} \int_{M_{t}}|h|^{2} \delta R .
$$

An application of the estimates of $T, \delta R$ then yields for $t, t^{\prime} \in\left[\tau_{0}, \tau\right]$

$$
\begin{aligned}
|h|_{g_{t}} \leq e^{A_{3}}|h|_{g_{t^{\prime}}}, \quad \int_{M_{t}}|h|^{2} \leq & e^{A_{3}} \int_{M_{t^{\prime}}}|h|^{2}, \\
& \text { with } A_{3}=\frac{a_{19}}{\gamma^{2} \tau_{0}^{n+1}} e^{a_{19} b_{1} d_{0}} b_{0} \sqrt[4]{\varepsilon} \sqrt{V_{0} .}
\end{aligned}
$$

We conclude from (1.43), (1.45), and Assumption III

$$
Q_{t_{0}} \geq \gamma_{0} A_{2} e^{-A_{3}} \int_{M_{t_{0}}}|h|^{2} \geq \frac{3 \gamma_{0}}{4} \int_{M_{t_{0}}}|h|^{2} .
$$

It follows that a neighborhood of $t_{0}$ is contained in $\Gamma$. Hence $\Gamma$ is open in $\left[\tau_{0}, \tau\right]$. But $\tau_{0} \in \Gamma$, therefore $\Gamma=\left[\tau_{0}, \tau\right]$ and the claim is proven.

In order to estimate $\lambda_{2 \beta_{0}}$ at time $\tau$, it remains to check whether (1.41) is implied by the following condition:

$$
\operatorname{tr}_{g_{\tau}} h=0, \quad\|h\|_{C^{0}\left(M_{\tau}\right)}^{2} \leq \frac{1}{2 \beta_{0}} \int_{M_{\tau}}|h|^{2} .
$$

Given an $h$ with (1.46), we put $h^{0}=h-\left(\operatorname{tr}_{g_{\tau_{0}}} h / n\right) g_{\tau_{0}}$. We compute

$$
\frac{\partial}{\partial t} \operatorname{tr}_{g} h=2\left(T+\frac{\delta R}{n} g\right) \cdot h .
$$

Using this, (1.45) and the condition $\operatorname{tr}_{g_{\mathrm{\tau}}} h=0$ one easily shows

$$
\left|\operatorname{tr}_{g_{\tau_{0}}} h\right|_{g_{\tau_{0}}} \leq A_{4}|h|_{g_{\tau}} \quad \text { with } A_{4}=e^{A_{3}} \frac{a_{20}}{\gamma^{2} \tau_{0}^{n+1}} e^{a_{20} b_{1} d_{0}} b_{0} \sqrt[4]{\varepsilon} \sqrt{V_{0}} .
$$

Hence, by a simple computation,

$$
\left\|h^{\circ}\right\|_{C^{0}\left(M_{\tau_{0}}\right)}^{2} \leq \frac{1}{\beta_{0}} \int_{M_{\tau_{0}}}\left|h^{0}\right|^{2} \text { and }\left|h^{0}\right|_{z_{\tau_{0}}} \geq \sqrt{\frac{6}{7}}|h| g_{\tau_{0}},
$$

provided the following is satisfied.

Assumption IV.

$$
\frac{1}{2} e^{2 A_{3}}\left(\frac{e^{A_{3}}+A_{4}}{1-A_{4} e^{A_{3}} / \sqrt{n}}\right) \leq 1, \quad 1-\frac{A_{4}}{\sqrt{n}} e^{A_{3}} \geq \sqrt{\frac{6}{7}} .
$$

By Lemma 4 we then have

$$
Q_{\tau_{0}}\left(h^{0}\right) \geq \gamma_{0} \int_{M_{\tau_{0}}}\left|h^{0}\right|^{2} .
$$


Hence, by an argument similar to that for the estimate (1.18) (plus the second inequality in (1.48)),

$$
Q_{\tau_{0}}\left(h^{0}\right) \geq \frac{5}{6} \gamma_{0} \int_{M_{\tau_{0}}}|h|^{2}+\frac{\gamma_{0}}{a_{21}} \int_{M_{\tau_{0}}}\left|\nabla h^{0}\right|^{2} .
$$

On the other hand,

$$
\left|\nabla \operatorname{tr}_{g_{\tau_{0}}} h\right| \leq a_{22}\left(\left|\nabla_{g_{\tau}}\right||h|+\left|g_{\tau}-g_{\tau_{0}}\right||\nabla h|\right),
$$

where the derivative and the norm refer to $g_{\tau_{0}}$. From (1.45) and the flow equation (0.2) we obtain

$$
\left|g_{\tau}-g_{\tau_{0}}\right| \leq \frac{a_{23}}{\gamma^{2} \tau_{0}^{n+1}} e^{A_{3}} e^{a_{23} b_{1} d_{0}} \cdot b_{0} \sqrt[4]{\varepsilon} \sqrt{V_{0}}
$$

while from (1.5) we derive

$$
\left|\nabla g_{\tau}\right| \leq \frac{a_{24}}{\gamma^{2} \tau_{0}^{n+1}} e^{2 A_{3}} e^{a_{24} b_{1} d_{0}} \cdot b_{0} \sqrt[4]{\varepsilon} \sqrt{V_{0}}
$$

Hence

$$
\begin{aligned}
\left|\nabla \operatorname{tr}_{t_{0}} h\right| \leq & A_{5}(|h|+|\nabla h|), \\
& \text { with } A_{5}=\frac{a_{22} \max \left(a_{23}, a_{24}\right)}{\gamma^{2} \tau_{0}^{n+1}} \cdot e^{2 A_{3}} e^{\max \left(a_{23}, a_{24}\right) b_{1} d_{0}} b_{0} \sqrt[4]{\varepsilon} \sqrt{V_{0}}
\end{aligned}
$$

Now we can estimate $Q_{\tau_{0}}(h)$ (under Assumption IV)

$$
\begin{aligned}
Q_{\tau_{0}}(h) \geq & Q_{\tau_{0}}\left(h^{0}\right)-a_{25}\left(1+\frac{1}{a}\right) \int_{M_{\tau_{0}}}\left(\left|\operatorname{tr}_{g_{\tau_{0}}} h\right|^{2}+\left|\nabla \operatorname{tr}_{g_{\tau_{0}}} h\right|^{2}\right) \\
& -a_{25} a \int_{M_{\tau_{0}}}\left(\left|h^{0}\right|^{2}+\left|\nabla h^{0}\right|^{2}\right) \\
\geq & Q_{\tau_{0}}\left(h^{0}\right)-a_{25}\left(\left(1+\frac{1}{a}\right)\left(A_{4}^{2} e^{2 A_{3}}+2 A_{5}+\frac{2 A_{5}^{2}}{\sqrt{n}-A_{5}}\right)\right. \\
& \left.+a\left(1+\frac{A_{4}}{\sqrt{n}} e^{A_{3}}\right)^{2}\right) \int_{M_{\tau_{0}}}|h|^{2} \\
& -a_{25}\left(\left(1+\frac{1}{a}\right) \frac{2 A_{5}^{2}}{\left(1-A_{5} / \sqrt{n}\right)^{2}}+a\right) \int_{M_{\tau_{0}}}\left|\nabla h^{0}\right|^{2},
\end{aligned}
$$

where $a>0$ is to be chosen. Now we choose $a=\left(\gamma_{0} / 2 a_{25}\right) \min \left(1 / a_{21}, 1 / 60\right)$ and make one more assumption.

\section{Assumption V.}

$$
\begin{gathered}
1+\frac{A_{4}}{\sqrt{n}} e^{A_{3} \leq \sqrt{2},} \quad a_{25}\left(1+\frac{1}{a}\right)\left(A_{4}^{2} e^{2 A_{3}}+2 A_{5}+\frac{2 A_{5}^{2}}{\sqrt{n}-A_{5}}\right) \leq \frac{\gamma_{0}}{60}, \\
a_{25}\left(1+\frac{1}{a}\right) \frac{A_{5}^{2}}{\left(1-A_{5} / \sqrt{n}\right)^{2}} \leq \frac{\gamma_{0}}{2 a_{21}} .
\end{gathered}
$$

Then we derive from the above estimate and the inequality (1.49) the desired condition (1.41), i.e.,

We summarize:

$$
Q_{\tau_{0}}(h) \geq \frac{4 \gamma_{0}}{5} \int_{M_{\tau_{0}}}|h|^{2}
$$


Lemma 5. Assume the same as in Lemma 4. Let $\tau \geq 2 \tau_{0}$ satisfy Condition $\mathrm{B}$ for some $B=\left(b_{0}, b_{1}, \gamma\right)$. Suppose that Assumptions I-V are all satisified. Then the estimates (1.28), (1.33), (1.34), (1.35), (1.36), (1.38), and (1.39) hold. Moreover, $\lambda_{2 \beta_{0}} \geq \frac{2}{3} \gamma_{0}$ holds at time $\tau$.

Now we are in a position to prove Theorem 1.

Proof of Theorem 1. Consider a Riemannian manifold $(M, g)$ of dimension $n \geq 3$ satisfying the condition $(\text { diam })^{2}\|R m\|_{C^{0}} \leq \Lambda,(\text { diam })^{2} \lambda_{e} \geq \sigma$. Dilating the metric we can achieve $\|R m\|_{C^{0}}=1$, diam $\leq \sqrt{\Lambda}, \lambda_{e} \geq \sigma / \Lambda$ (we omit the trivial case $\|R m\|_{C^{0}}=0$ ). It suffices to show that the dilated metric (which will be denoted by $g_{0}$ ) can be deformed to an Einstein metric through the Ricci flow. Consider the Ricci flow starting at $g_{0}$. On account of Lemma 2, Lemma 4 , and estimate $(1.13)$ we have on $\left[0, \tau_{0}\right]:\|R m\|_{C^{0}} \leq 2, \lambda_{\beta} \geq \gamma_{0}$, and $\operatorname{diam} M_{t} \leq \sqrt{c_{11}} d_{0} \quad\left(\beta=4 \beta_{0}\right)$. From Lemma 2 and equation (6) in Lemma 1 we also derive for $t \in[0, \tau]$ the estimate $\int_{M_{t}}|T|^{2} \leq a_{26} \int_{M_{0}}|T|^{2}$ for some $a_{26}=a_{26}(n)$, which implies $\int_{M_{t}}|T|^{2} \leq a_{26} e e^{-\gamma_{0} t / 4} \int_{M_{0}}|T|^{2}$. We conclude that $\tau_{0}$ satisfies Condition $\mathrm{B}_{0}$ with $B_{0}=\left(2 a_{26} e, 2 \sqrt{c_{11}}, \gamma_{0} / 4\right)$. Define $\Gamma=$ $\left\{t \geq \tau_{0}: t\right.$ satisfies Condition $\left.\mathrm{B}\right\}$. Now we choose a sufficiently large number $a_{26}=a_{26}(n)$ such that the condition

$$
\left(a_{26} / \gamma_{0}^{4} \tau_{0}^{3 n}\right) e^{a_{26} d_{0}} \sqrt[8]{\varepsilon} \leq 1
$$

implies Assumptions I-V with $b_{0}=2 a_{26} e, b_{1}=2 \sqrt{c_{11}}$, and $\gamma=\frac{1}{4} \gamma_{0}$. This is possible because of estimate (1.33), standard volume comparisons and the algebraic forms of these assumptions. Next we choose a suitable number $c=$ $c(n)$ such that $(1.51)$ is implied by the condition $d_{0} \leq \sqrt{\Lambda}, \lambda_{e} \geq \sigma / \Lambda$, and

$$
\varepsilon \leq(1 / c) e^{-c \sqrt{\Lambda}} \min \left(1,(\sigma / \Lambda)^{8(3 n+8)}\right)
$$

Now if the metric $g$ satisfies the condition

$$
f|T|^{2} \leq \frac{1}{c} e^{-c \sqrt{\Lambda}} \min \left(1,\left(\frac{\sigma}{\Lambda}\right)^{8(3 n+8)}\right)\|R m\|_{C^{0}}^{2},
$$

then (1.52) is true for the dilated metric $g_{0}$. From Lemma 5 we then derive that $\Gamma$ is closed. We also infer that condition (i) in Condition $B_{0}$ is an open condition. Since the remaining conditions are obviously open, we conclude that $\Gamma$ is open in $\left[\tau_{0}, \infty\right)$. Thus $\Gamma=\left[\tau_{0}, \infty\right)$. It follows that the Ricci flow exists for all time and converges exponentially as $t$ goes to $\infty$. (Convergence in $C^{2}$ is immediate from estimates (1.28), (1.33), (1.34), and (1.35). Higher order convergence follows readily.) Taking limit in the flow equation $(0.2)$ we see that the limit metric is Einstein. This finishes the proof of the second pinching case of the theorem. To treat the first pinching condition, we dilate the metric to achieve diam $=1,\|R m\|_{C^{0}} \leq \Lambda$ and $\lambda_{e} \geq \sigma$. Arguing essentially in the same way as before we then reach the conclusion. (Some modifications are in order. For example, one first derives from Lemma 4: if the initial metric satisfies $\|R m\|_{C^{0}} \leq \Lambda$ and $\lambda_{e}>0$, then on the time interval $\left[0, a(n) \lambda_{e}^{2} / \Lambda\left(1+\lambda_{e}^{2}\right)\right]$ of the Ricci flow, the estimate $\lambda_{\beta_{0} / \Lambda^{2}} \geq \Lambda \lambda_{\varepsilon} / c(n)\left(1+\lambda_{\ell}\right)$ holds.

Comments on Remarks 1 and 2 . Remark 2 can be proven in the same way as above. To justify Remark 1, we apply a more general version of Lemma 3 , in which the constant $b$ is replaced by an $L^{p / 2}$ function. This version is 
formulated as Theorem 4.1 in [Ya]. As mentioned before, one has to correct its statement suitably.

Proof of Corollary to Theorem 1. Deformation to Einstein metrics is immediate from Remark 2 and Proposition 4. A negative upper bound for $R$ can be deduced from the pointwise maximum principle applied to equation (4) in Lemma 1 (together with decay estimates for $|T|$ and $\delta R$ ). This combined with the decay estimate for $|T|$ implies the claimed negativity of Ricci curvatures.

\section{STABILITY AND RIEMANN PINCHING}

Stability of Einstein metrics is usually defined in terms of the second variation of the normalized total scalar curvature functional $\mathbf{S}$ in directions which are transversal (i.e., divergence free) traceless symmetric 2-tensors, see [Ko3]. Our concept of stability (in the special case of Einstein metrics) is actually equivalent to this.

Proposition 1. An Einstein metric $g$ is stable (in the sense of Definition 1) if and only if $\left.\left(d^{2} / d t^{2}\right) \mathbf{S}(g+t h)\right|_{t=0}<0$ for all nonzero transversal traceless symmetric 2-tensors $h$.

Proof. Following the computations in [ ] we derive for an arbitrary traceless symmetric 2-tensor $h$

$$
\left.\frac{d^{2}}{d t^{2}} \mathbf{S}(g+t h)\right|_{t=0}=-\int h \cdot \tilde{L} h,
$$

where

$$
(\tilde{L} h)_{i j}=-\frac{1}{2} \Delta h+\frac{1}{2} g^{k l}\left(h_{i k ; j l}+h_{j k ; i l}\right)-\frac{1}{n} R h_{i j}
$$

(assuming w.l.o.g. $V(g)=1$ ). An application of the Ricci identity then yields

$$
\left.\frac{d^{2}}{d t^{2}} \mathbf{S}(g+t h)\right|_{t=0}=-\frac{1}{2} Q(h)+\int|\delta h|^{2}
$$

where $\delta h$ is the divergence of $h,(\delta h)_{i}=g^{i k} h_{i j ; k}$. For convenience let us denote the second variation $\left.\left(d^{2} / d t^{2}\right) \mathbf{S}(g+t h)\right|_{t=0}$ by $\tilde{Q}(h)$. From $(2.1)$ the "only if" part of the proposition follows immediately. Now assume $\widetilde{Q}(h)<0$ for all nonzero transversal traceless symmetric 2-tensors $h$. If $g$ is a round sphere metric, then $\lambda_{e}>0$ according to Proposition 3 below. Otherwise, for any traceless symmetric 2-tensor $h$ we have the orthogonal decomposition $h=$ $h^{0}+h^{1}$, where $h^{1}$ is transversal traceless and $h^{0}$ is a Lie derivative of $g$ (see [Be, 4.57]). Then $\widetilde{Q}(h)=\widetilde{Q}\left(h^{1}\right)$ and hence $Q(h)=-2 \widetilde{Q}\left(h^{1}\right)+2 \int|\delta h|^{2}$ by (2.1). It follows $Q(h)>0$, provided that $h$ is not identically zero.

Now we present some geometric conditions which imply stability (of a metric which is not necessarily Einstein). Stability of Einstein metrics has been studied by Koiso [Ko1, Ko2, Ko3]. We largely follow his arguments. We denote respectively by $\alpha_{0}, \alpha_{0}^{\prime}$ the maximum eigenvalues of the operators $h \mapsto R m(h)+R h / n$ and $h \mapsto R m(h)$ acting on traceless symmetric 2-tensors $h$.

Proposition 2. We have $\lambda_{e} \geq-\min \left(\alpha_{0}, \alpha_{0}^{\prime}\right)$. Hence stability is implied by the condition $\alpha_{0}<0$ or $\alpha_{0}^{\prime}<0$. 
Proof. As in the proof of Theorem 2.5 in [Ko2], one gets, by computing $\int \sum_{i, j, k}\left(h_{i j ; k}-h_{j k ; i}\right)^{2}$,

$$
\int|\nabla h|^{2}-\int R m(h) \cdot h \geq \int|\delta h|^{2}-\frac{1}{n} \int R|h|^{2}-\int T(h) \cdot h,
$$

hence $\lambda_{e} \geq-\alpha_{0}$. The estimate $\lambda_{e} \geq-\alpha_{0}^{\prime}$ is trivial.

Proposition 3. Sufficiently (Riemann) pinched metrics of nonzero scalar curvature are stable. More precisely, we have

(1)

$$
\begin{aligned}
\lambda_{e} & \geq \max \left(\frac{2 \bar{R}}{n(n-1)}-2(\sqrt{n}+1)\left\|\widetilde{R}_{m}\right\|_{C^{0}},-\frac{n-1}{n(n-1)} \bar{R}-2\|\widetilde{R} m\|_{C^{0}}\right) \\
& \geq \frac{1}{n(n-1)}|\bar{R}|-2(\sqrt{n}+1)\|\widetilde{R} m\|_{C^{0}},
\end{aligned}
$$

$$
\lambda_{e} \geq \max \left(\min _{M}\left(\frac{2 R}{n(n-1)}-2|\stackrel{\circ}{R} m|-|T|\right), \min _{M}\left(-\frac{n-2}{n(n-1)} R-|\stackrel{\circ}{R} m|\right)\right) .
$$

(The first relates to global pinching, the second to pointwise pinching.)

Proof. These are simple algebraic computations, applying Proposition 2. The first entry in $\max \left(\right.$ ) (of either estimate) comes from an estimate of $\alpha_{0}^{\prime}$, while the second from that of $\alpha_{0}$.

Proposition 4. Partially Ricci pinched metrics of negative sectional curvatures are stable. More precisely, we have

$$
\lambda_{e} \geq-(n-2) K^{+}-\max _{M}(r-R / n),
$$

where $r$ denotes Ricci curvatures.

Proof. Consider the operator $h \mapsto R m(h)$ (acting on traceless symmetric 2tensor $h$ ) at a fixed point. Choose an orthonormal frame in which $h$ is diagonalized. Then $\operatorname{Rm}(h) \cdot h=\sigma_{i j} h^{i} h^{j}$, where $h^{i}=h^{i i}$ and $\sigma_{i j}=R_{i j i j}$ (no summation in this equation). Let $x$ be an eigenvector of the matrix $\left(\sigma_{i j}\right)$ belonging to an eigenvalue $\lambda$ such that $\sum_{i=1}^{n} x_{i}=0$. W.l.o.g. we assume $x_{n}=\max _{1 \leq i \leq n}\left|x_{i}\right|$. Then

$$
\begin{aligned}
\lambda x_{n} & =\sum_{j=1}^{n} \sigma_{n j} x_{j}=-\sum_{j=1}^{n} \sigma_{n j} x_{n}+\sum_{j=1}^{n} \sigma_{n j}\left(x_{j}+x_{n}\right) \\
& \leq-r_{n} x_{n}+K^{+} \sum_{j \neq n}\left(x_{j}+x_{n}\right),
\end{aligned}
$$

where $r_{n}=\sum_{j=1}^{n} \sigma_{n j}$. But $\sum_{j \neq n}\left(x_{j}+x_{n}\right)=(n-2) x_{n}$. Consequently $\lambda \leq$ $-r_{n}+(n-2) K^{+}$, which implies $\alpha_{0} \leq(n-2) K^{+}+R / n-r_{n}$ and hence the desired estimate by Proposition 2.

To deal with Riemann pinching, we need a few more evolution equations. 
Lemma 6. Along the Ricci flow we have

$$
\frac{\partial}{\partial t} \bar{R}=2 f|T|^{2}+\left(\frac{2}{n}-1\right) f(\delta R)^{2}
$$

$$
\frac{\partial}{\partial t}(\delta R)=\Delta(\delta R)+2|T|^{2}+\frac{2 \delta R}{n} R-2 f|T|^{2}+\left(1-\frac{2}{n}\right) f(\delta R)^{2}
$$

(3) $\frac{\partial}{\partial t} \int(\delta R)^{2}=-2 \int|\nabla R|^{2}+4 \int \delta R|T|^{2}-\left(1-\frac{4}{n}\right) \int(\delta R)^{3}+\frac{4 \bar{R}}{n} \int(\delta R)^{2}$,

$$
\frac{\partial}{\partial t}|\stackrel{\circ}{R} m|^{2}=\Delta|\stackrel{\circ}{R} m|^{2}-2|\nabla \stackrel{\circ}{R} m|^{2}-\frac{4 R}{n}|\stackrel{\circ}{R} m|^{2}+P,
$$

where

$$
P=\frac{16}{n(n-1)} R|T|^{2}-8\left(\stackrel{\circ}{R}_{p i j q} \stackrel{\circ}{R}_{k l}^{q} \stackrel{\circ}{R}^{i j k l}+\stackrel{\circ}{R}_{p i k q} \stackrel{\circ}{R}_{j l}^{q} \stackrel{\circ}{R}^{i j k l}\right) .
$$

Proof. Equations (1), (2), and (3) are easy computations. The last one follows from Lemma 3 in [Ni] and Lemma 17.1 in [Hal].

Proof of Theorem 2. According to [Gr], conditions (2) and (3) can be reduced to (1). So let us fix $n, C$ and assume $d \leq C,-1 \geq K^{+} \geq K^{-} \geq-1-\varepsilon$, where $\varepsilon>0$ is to be determined. It is immediate from Theorem 1 and Proposition 3 that choosing $\varepsilon$ sufficiently small we can obtain Ricci deformation to Einstein manifolds. We show that pinching is preserved in the limit. Applying the pointwise maximum principle to equation (4) in Lemma 6 we infer

$$
\|\stackrel{\circ}{R} m\|_{C^{0}\left(M_{t}\right)} \leq C_{1}(n)\|\stackrel{\circ}{R} m\|_{C^{0}\left(M_{0}\right)}, \quad 0 \leq t \leq C_{2}(n) .
$$

$\left(C_{1}, C_{2}, \ldots\right.$ are all positive constants.) Setting $G_{i j k l}=g_{i k} g_{j l}-g_{i l} g_{j k}$ we compute

$$
\left.\frac{\partial}{\partial t}|\stackrel{\circ}{R} m|^{2}=\dot{\langle} \stackrel{\circ}{R} m, \stackrel{\circ}{R} m\right\rangle+2\left\langle\stackrel{\circ}{R} m, \dot{R} m-\frac{\dot{R}}{n(n-1)} G-\frac{R}{n(n-1)} \dot{G}\right\rangle .
$$

$\dot{R} m$ can be handled with (1.6), while the other terms are easy to compute. Employing the estimates (1.33), (1.34), (1.35), and (1.36) with suitable constants (these estimates hold by the proof of Theorem 1) we then deduce for $t \geq C_{2}(n)$

$$
\frac{\partial}{\partial t}|\stackrel{\circ}{R} m|^{2} \leq C_{3}(n, C)\left(\int_{M_{0}}|T|^{2}\right)^{1 / 8} e^{-C_{4}(n) t} .
$$

Integrating this inequality and appealing to (2.2) yield for $0 \leq t \leq+\infty$

$$
\|\stackrel{\circ}{R} m\|_{C^{0}\left(M_{t}\right)} \leq C_{4}(n, C)\left(\|\stackrel{\circ}{R} m\|_{C^{0}\left(M_{0}\right)}+\sqrt[8]{\|\stackrel{\circ}{R} m\|_{C^{0}\left(M_{0}\right)}}\right)
$$

hence pinching is preserved along the flow and in the limit. We claim that $M_{\infty}$ is a space form, provided that $\varepsilon$ has been chosen small enough. Otherwise, we would have a sequence of limit Einstein manifolds $M_{\infty}^{k}$, none of which is a space form, such that their volumes are uniformly bounded away form zero (by Margulis-Heintze theorem [Gr] and the fact that Ricci flow preserves volume), their diameters are uniformly bounded from above (by estimate (1.38)), their sectional curvatures are uniformly bounded in absolute value (by (1.36)), and 
that $\|\stackrel{\circ}{R} m\|_{C^{0}\left(M_{\infty}^{k}\right)} \rightarrow 0$ as $k \rightarrow \infty$. By Gromov's compactness theorem [Pe], a subsequence of $M_{\infty}^{k}$ converges to a $C^{1, \alpha}$-Riemannian manifold $M_{\infty}^{\infty}$ in the $C^{1, \alpha}$-topology. But $M_{\infty}^{k}$ are Einstein, hence in harmonic coordinates we have higher order estimates (this is a simple PDE, see, e.g., [De-Ka]). It follows that this subsequence actually converges smoothly and $M_{\infty}^{\infty}$ is a space form. But space forms are isolated Einstein metrics (under constant volume condition) because they are stable (Proposition 3), hence we arrive at a contradiction.

It remains to show that negative curvature is preserved along the flow and in the limit. From equation (2) in Lemma 6 we derive

$$
\begin{aligned}
\frac{\partial}{\partial t}(\delta R)^{2}= & \Delta(\delta R)^{2}-2|\Delta R|^{2}+2 \delta R|T|^{2}+\frac{2(\delta R)^{2}}{n} R \\
& -2 \delta R f|T|^{2}+\left(1-\frac{2}{n}\right) \delta R f(\delta R)^{2} .
\end{aligned}
$$

By the estimate (1.36) this implies

$$
\frac{\partial}{\partial t}(\delta R)^{2} \leq \Delta(\delta R)^{2}+C_{5}(n) \max _{M_{t}}(\delta R)^{2}+C_{5}(n) \max _{M_{t}}|T|^{2} .
$$

The pointwise maximum principle applied to this equation yields for $t \geq 0$

$$
\max _{M_{t}}(\delta R)^{2} \leq e^{C_{5}(n) t}\left(\max _{M_{0}}(\delta R)^{2}+\int_{0}^{t} \max _{M_{t}}|T|^{2} d t\right) .
$$

This together with (2.5) provides an estimate of $\delta R$ on the time interval $[0,1]$. For $t \geq 1$ we can estimate $\delta R$ in terms of (1.33). We conclude that $\delta R$ remains small (in dependence of $\varepsilon$ ) along the flow. On the other hand, equation (1) in Lemma 6 together with the estimate (2.5) implies $\bar{R} \leq-\frac{1}{2}$ (assuming again that $\varepsilon$ has been chosen small enough). This, the smallness of $\delta R$ and the curvature pinching together clearly imply that sectional curvatures remain negative along the flow and in the limit.

Proof of Theorem 4. First rescale the metric to achieve $\|R m\|_{C^{0}}=1$. Then the Ricci flow exists at least on the interval $[0, \tau(n)]$ according to Lemma 2. From equation (4) in Lemma 6 we obtain a parabolic inequality for $|\stackrel{R}{R} m|$, to which we apply Lemma 3 . We arrive at

$$
\|\stackrel{\circ}{R} m\|_{C^{0}\left(M_{t}\right)}^{2}<C_{1}(n, \Lambda) \varepsilon \bar{R}_{0}^{4}, \quad \tau(n) / 2 \leq t \leq \tau(n),
$$

where $\bar{R}_{0}$ denotes $\bar{R}$ at $t=0$. But equation (1) in Lemma 6 implies $\bar{R} \leq$ $\bar{R}_{0}+2 f_{0}^{t} d t \int|T|^{2} \leq \bar{R}_{0}-C_{2}(n) \varepsilon \bar{R}_{0} \leq \frac{1}{2} \bar{R}_{0}$, provided that $\varepsilon \leq 1 / 2 C_{2}(n)\left(\int|T|^{2}\right.$ is estimated by using equation (6) in Lemma 1). Hence

$$
\|\stackrel{\circ}{R} m\|_{C^{0}<-2 C_{1}(n, \Lambda) \varepsilon \bar{R} \text { on }[\tau(n) / 2, \tau(n)] .}
$$

On the other hand, by the arguments which lead to the estimates (1.33) we can deduce

$$
\|\delta R\|_{C^{0}} \leq-C_{2}(n, \Lambda) \sqrt[4]{\varepsilon} \bar{R} \text { on }[\tau(n) / 2, \tau(n)] .
$$

Now consider the time $t_{0}=\tau(n) / 2$. By Lemma 2 and the estimate (1.13) we have at $t_{0}:(\operatorname{diam})^{2}\|R m\|_{C^{0}} \leq C_{3}(n) \Lambda$. We dilate the metric $g_{t_{0}}$ to achieve 
$\|R m\|_{C^{0}}=1$. It suffices to show that the Ricci flow starting at the dilated metric $\tilde{g}_{t_{0}}$ will converge to a hyperbolic space form, because Ricci flow is scale invariant in the following sense: if $\frac{\partial g}{\partial t}=-2 R c_{g}+\frac{2 \bar{R}_{g}}{n} g$ and we set $\tilde{g}=c g$, $\tilde{t}=c t$, then

$$
\frac{\partial \tilde{g}}{\partial t}=-2 R c_{\tilde{g}}+\frac{2 \bar{R}_{\tilde{g}}}{n} \tilde{g}
$$

The estimates (2.10) and (2.11) are retained after the dilation. They imply $\|R m\|_{C^{0}} \leq C_{3}(n) \bar{R} \quad\left(\varepsilon\right.$ being chosen small enough) and hence $\bar{R} \leq-C_{3}(n)^{-1}$. Dilating the metric once again and choosing $\varepsilon$ sufficiently small, we can achieve the condition for deformation in Theorem 2 and therewith we are done.

Proof of Theorem 4' $4^{\prime}$ This is basically the same as above. Again rescale to get $\|R m\|_{C^{0}}=1$. But instead of (2.9) we use

$$
\|\stackrel{\circ}{R} m\|_{C^{0}}^{2} \leq C_{1}(n, \Lambda, k) \varepsilon \bar{R}_{0}^{4}, \quad \text { on }[\tau(n) / k, \tau(n)], k=1,2, \ldots
$$

Furthermore, from equation (3) in Lemma 6 and the assumption we see $\int(\delta R)^{2}$ $\leq C_{2}(n, C) \bar{R}_{0}^{2}$ on $[0, \tau(n)]$ (choosing $\varepsilon$ small). From this and equation (4) in Lemma 6 we then derive $\left|\bar{R}-\bar{R}_{0}\right| \leq C_{3}(n, C) \bar{R}_{0} t, t \in[0, \tau(n)]$. Combining this inequality with $(2.12)$ yields

$$
\|\stackrel{\circ}{R} m\|_{C^{0}}<C_{4}(n, C, \Lambda) \varepsilon \bar{R}, \quad \text { at } t_{0}=\frac{1}{2} C_{3}(n, C)^{-1} .
$$

In analogy to $(2.11)$ we obtain at $t_{0}:\|\delta R\|_{C^{0}} \leq C_{5}(n, C, \Lambda) \sqrt[4]{\varepsilon} \bar{R}$. Now we can rescale to achieve $1+C_{6}(n, C, \Lambda) \sqrt[4]{\varepsilon} \geq K^{+} \geq K^{-} \geq 1$ and diam $\leq C_{7}(n) \sqrt{\Lambda}$. Applying Theorem 1 and Proposition 3 we then achieve Ricci deformation to an Einstein manifold. The arguments in the proof of Theorem 2 can be adapted to show that the limit is actually a spherical space form.

Proof of Theorem 3. This is similar to and easier than the proof of Theorems 4 and $4^{\prime}$. One notes that the pinching $f|\widetilde{R} m|^{2} \leq \varepsilon \bar{R}^{2}$ implies $f|\delta R|^{2} \leq C(n) \varepsilon \bar{R}^{2}$ for some $C(n)$.

Proof of Theorem 5. The proofs of Lemma 5 and Theorem 1 (combined with the stability estimate Proposition 3 ) basically apply here but two changes are necessary. The first is that instead of the second inequality in Lemma 3 we should now use the first one everywhere. For the application of this inequality we notice that the Margulis-Heintze theorem [Gr] yields an upper bound for $V^{-2 n}$, while the relation $C_{S} \leq a(n) C_{I}^{2 / n}$ (for some $a(n) \geq 1$, see [Li]) provides an estimate for the Sobolev constant $C_{S}$ of the initial metric (we consider $C_{I}$ as a more basic concept than $C_{S}$ ). We shall see below how to control the Sobolev constant along the flow. The second change is that we have to estimate $\delta R$ in a new way. The previous estimation of $\delta R$ (contained in (1.33) and used elsewhere) was done with the help of the inequality $|\delta R| \leq($ diam $) \max |\nabla R|$, but we have no control over diam now.

To appeal to the previous arguments, we again dilate the initial metric to achieve $\|R m\|_{C^{0}}=1$. Then the pinching is changed to $-c(n) \geq K^{+} \geq K^{-} \geq$ $-c(n)-\tilde{c}(n) \varepsilon$ for some $c(n)>0, \tilde{c}(n)>0$, whereas the Sobolev constant remains the same. Now we define a new type of Condition $B$. We say that a finite time $\tau \geq \tau_{0}$ satisfies Condition $\widetilde{\mathrm{B}}$ for $\widetilde{B}=\left(b_{0}, \gamma, K, C, a\right)$ obeying 
$b_{0} \geq 1,0<\gamma \leq \gamma_{0} / 4,-n(n-1) c(n) / 2 \geq K \geq-2 n(n-1) c(n), C>0$, and $0<a \leq(n-1) c(n)$, if the following is true:

(i) $\lambda_{\beta}>\gamma_{0} / 2$ on $[0, \tau]$, with $\beta=4 \int_{M_{0}}|T|^{2}$,

(ii) $\|R m\|_{C^{0}}<10$ on $[0, \tau]$,

(iii) $\int_{M_{t}}|T|^{2}<b_{0} e^{-\gamma t} \int_{M_{0}}|T|^{2}$ for $t \in[0, \tau]$,

(iv) $\bar{R}<K$ on $[0, \tau]$,

(v) $C_{S}^{(2)}<C$ on $[0, \tau]$,

(vi) $\|\delta R\|_{C^{0}}<a$ on $[0, \tau]$.

Again we derive suitable estimates from Condition $\widetilde{B}$ in order to show that it is an open and closed condition, whenever the pinching number $\varepsilon$ is small enough and $\widetilde{B}$ is suitably chosen. We indicate how to estimate $C_{S}$ and $\delta R$.

Applying (iii), (iv), and (vi) to equation (3) in Lemma 6 we derive

$$
\frac{\partial}{\partial t} \int(\delta R)^{2} \leq 4 a b_{0} e^{-\gamma t} \int_{M_{0}}|T|^{2}+(n-1) c(n) K \int(\delta R)^{2}
$$

for $t \in[0, \tau]$ (with $\tau$ satisfying Condition $\widetilde{\mathbf{B}}$ for some $\widetilde{B}$ ). Setting $\bar{\gamma}=$ $\min \left(\frac{1}{2} \gamma,(n-1) c(n)|K|\right)$ we then have for $t \in[0, \tau]$

$$
\int_{M_{t}}(\delta R)^{2} \leq e^{-\bar{\gamma} t}\left(\int_{M_{0}}(\delta R)^{2}+\frac{8 a b_{0}}{\gamma} \int_{M_{0}}|T|^{2}\right) .
$$

Now we can apply Lemma 3 to (2.6) to deduce an exponential decay estimate for $\|\delta R\|_{C^{0}}$ (noting an estimate similar to (1.28) in dependence of $C$ instead of $b_{1}$ and $d_{0}$ ).

Next we compute for an arbitrary smooth function $f$ and $p>0$

$$
\left.\left.\left|\frac{d}{d t} \int\right| f\right|^{p}\left|\leq \max _{M_{t}}\right| \delta R\left|\int\right| f\right|^{p},
$$

hence

$$
e^{-\int_{0}^{t} \max _{M_{t}}|\delta R| d t} \int_{M_{0}}|f|^{p} \leq \int_{M_{t}}|f|^{p} \leq e^{\int_{0}^{t} \max _{M_{t}}|\delta R| d t} \int_{M_{0}}|f|^{p}
$$

On the other hand,

$$
\left.\left.\left|\frac{d}{d t} \int\right| \nabla f\right|^{2}\left|\leq\left(\left(1+\frac{4}{n}\right) \max _{M_{t}}|\delta R|+4 \max _{M_{t}}|T|\right) \int\right| \nabla f\right|^{2},
$$

which yields an estimate for $\int|\nabla f|^{2}$ similar to (2.15). Combining these two estimates we conclude

$$
\left(\int_{M_{t}}|f|^{2 n /(n-2)}\right)^{(n-2) / n} \leq A(\tau)\left(C_{S}\left(M_{0}\right) \int_{M_{t}}|\nabla f|^{2}+V^{-2 / n} \int_{M_{\tau}} f^{2}\right),
$$

are $A(\tau)=\exp \left(\int_{0}^{\tau}\left(\|\delta R\|_{C^{0}}+\|T\|_{C^{0}}\right) d t\right)$. This implies an estimate for $C_{S}^{(2)}\left(M_{\tau}\right)$, provided that the pinching number $\varepsilon$ has been chosen small enough.

Other quantities can be estimated along the lines of arguments in the proof of Lemma 5. We let the reader complete the proof. Note that the arguments in the proof of Theorem 2 can be applied to show negativity of curvature along the flow and the desired pinching in the limit. 
Proof of Theorem 6. Rescale the metric to get $V=1$. Arguing in a fashion similar to the proof of Theorem 4, we can reduce to Theorem 5. Since an upper bound for volume is available this time, we can actually achieve deformation to space forms also in dimensions $\geq 4$; cf. the proof of Theorem 2 .

A remark on pinched Einstein manifolds. Applying equation (4) in Lemma 6 to an Einstein metric $g$ we obtain

$$
\Delta|\stackrel{\circ}{R} m|^{2}-2|\nabla \stackrel{\circ}{R} m|^{2}-(4 R / n)|\stackrel{\circ}{R} m|^{2}+P=0 .
$$

Since $T \equiv 0, P$ is cubic in $\stackrel{\circ}{R} m$. Hence, if $R>0$ and $|\stackrel{\circ}{R} m| \leq \varepsilon R$ for $\varepsilon=\varepsilon(n)$ sufficiently small, then by the maximum principle we conclude $\stackrel{\circ}{R} m \equiv 0$, and consequently $g$ has constant sectional curvatures. A good calculation of $\varepsilon$ can be found in [Hu] or [Ma]. From [Ha2] we can also conclude: 4-dimensional Einstein manifolds of positive curvature operator must be space forms.

\section{REFERENCES}

[Be] A. Besse, Einstein manifolds, Springer, 1987.

[De-Ka] D. DeTurck and J. Kazdan, Some regularity theorems in Riemannian geometry, Ann. Sci. École Norm. Sup. (4) 14 (1980), 249-260.

[Fa-Jo] F. T. Farrell and L. E. Jones, Negatively curved manifolds with exotic smooth structures, J. Amer. Math. Soc. 2 (1989), 899-908.

[Gal] S. Gallot, Inégalités isopérimétriques, courbure de Ricci et invariants géométriques. I, C. R. Acad. Sci. Paris Ser. I Math. 296 (1983), 333-336.

[Gao] L. Gao, Convergence of Riemannian manifolds, Ricci pinching and $L^{N / 2}$-curvature pinching, preprint.

[Gr] M. Gromov, Manifolds of negative curvature, J. Differential Geom. 13 (1978), 223-230.

[Gr-Th] M. Gromov and W. Thurston, Pinching constants for hyperbolic manifolds, Invent. Math. 89 (1987), 1-12.

[Ha1] R. S. Hamilton, Three-manifolds with positive Ricci curvature, J. Differential Geom. 17 (1982), 255-306.

[Ha2] _ Four-manifolds with positive curvature operator, J. Differential Geom. 24 (1986), 153179.

[Ha3] _- Lectures on heat equations in geometry, Lecture Notes, Hawaii, 1989.

[Hu] G. Huisken, Ricci deformation of the metric on a Riemannian manifold, J. Differential Geom. 17 (1985), 47-62.

[Ko1] N. Koiso, Nondeformability of Einstein metrics, Osaka J. Math. 15 (1978), 419-433.

[Ko2] __, On the second derivative of the total scalar curvature, Osaka J. Math. 16 (1979), 413-421.

[Ko3] _ Rigidity and stability of Einstein metrics. The case of compact symmetric spaces, Osaka J. Math. 17 (1970), 51-73.

[Li] P. Li, On the Sobolev constant and the p-spectrum of a compact Riemannian manifold, Ann. Sci. École Norm. Sup. 13 (1980), 451-469.

[Ma] C. Margerin, Pointwise pinched manifolds are space forms, Proc. Sympos. Pure Math., vol. 44, Amer. Math. Soc., Providence, R.I., 1986, pp. 307-328.

[Mi] M. Min-Oo, Almost Einstein manifolds of negative Ricci curvature, J. Differential Geom. 32 (1990), 457-472.

[Mi-Ru] M. Min-Oo and E. A. Ruh, $L^{2}$-curvature pinching, preprint.

[Mo] J. Moser, A Harnack inequality for parabolic differential equations, Comm. Pure Appl. Math. 17 (1964), 101-134. 
[Ni] S. Nishikawa, Deformation of Riemannian metrics and manifolds with bounded curvature ratios, Proc. Sympos. Pure Math., vol. 44, Amer. Math. Soc. Providence, R.I., 1986, pp. 343-352.

[Pe] S. Peters, Convergence of Riemannian manifolds, Composito Math. 62 (1987), 3-16.

[Ru] E. A. Ruh, Riemannian manifolds with bounded curvature ratios, J. Differential Geom. 17 (1982), 643-653.

[Sc] R. Schoen, Variational theory for the total scalar curvature functional for Riemannian metrics and related topics, preprint.

[Sh] W. Shi, Ricci deformation of the metric on complete noncompact Riemannian manifolds, J. Differential Geom. 29 (1989).

[Ya] D. Yang, $L^{p}$ pinching and compactness theorems for compact Riemannian manifolds, preprint.

Department of Mathematics, Stanford University, Stanford, California 94305

Current address: Department of Mathematics, University of California, Santa Barbara, California 93106

E-mail address: yer@math.ucsb.edu 